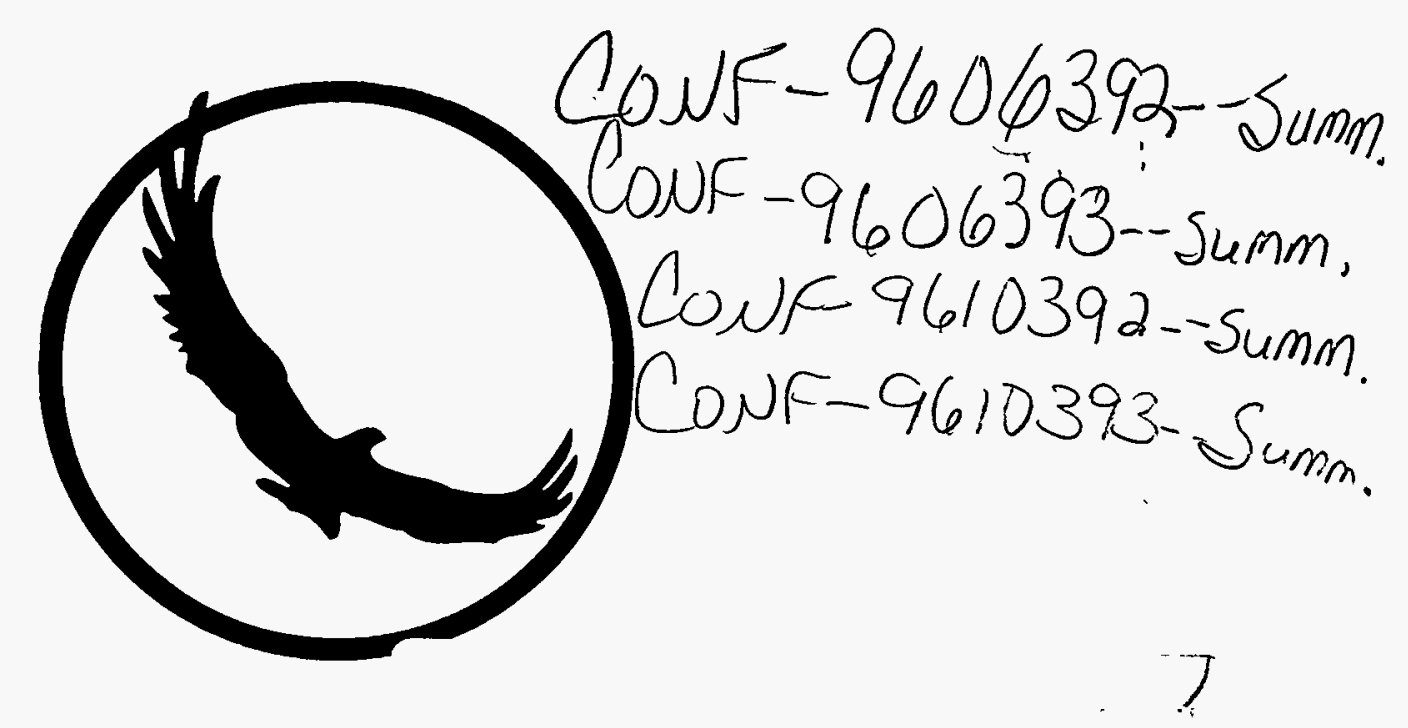

\title{
The Keystone Center
}

U.S. Department of Energy

Environmental Management Science Program

Final Technical Report

Grant \# DE-FG03-96ER62220

January 22, 1998

DISTRBSTTON OF THIS DOCUMENT IS UNLLMTIED

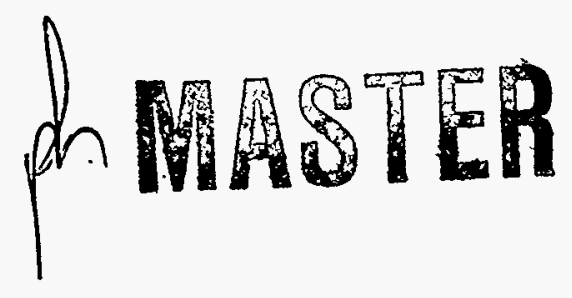

THE KEYSTONE CENTER

P.O. Box 8606, Keystone, Colorado 80435-7998

Phone 970/468-5822 Fax 970/262-0152 Email tkcspp@keystone.org 


\section{DISCLAIMER}

This report was prepared as an account of work sponsored by an agency of the United States Government. Neither the United States Government aor any agency thereof, nor any of their employees, makes any warranty, express or implied, or assumes any legal liability or responsibility for the accuracy, completeness, or usefulness of any information, apparatus, product, or process disclosed, or represents that its use would not infringe privately owned rights. Reference herein to any specific commercial product, process, or service by trade name, trademark, manufacturer, or otherwise does not necessarily constitute or imply its endorsement, recommendation, or favoring by the United States Government or any agency thereof. The views and opinions of authors expressed herein do not necessarily state or reflect those of the United States Government or any agency thereof. 


\section{DISCLAIMER}

Portions of this document may be illegible electronic image products. Images are produced from the best available original document. 


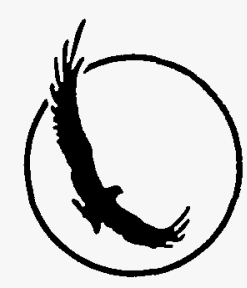

\section{The Keystone Center}

\section{Memorandum}

To: Jay Dodson, Program Acquisition and Assistance Division, U.S. Department of Energy

From: $\quad$ Kevin Curtis and Alyssa Wittenborn

Subject: $\quad$ Final Technical Report - Grant \# DE-FG03-96ER62220

Date: $\quad$ January 22, 1998

This memorandum shall serve as the Final Technical Report from The Keystone Center to the Department of Energy for our work with the Environmental Management Science Program (EMSP). This memorandum will describe briefly the various activities in which The Keystone Center engaged with the EMSP under Grant \# DE-FG03-96ER62220. The reports resulting from these activities, including four meeting summaries and a Final Report on Assessment Activities for EMSP, are attached for more detailed information.

The Keystone Center began its work with the EMSP in May, 1996, when The Center agreed to design, organize, and facilitate stakeholder meetings at two DOE sites: Savannah River and Hanford. These meetings were held in June, 1996 with the purposes of introducing the EMSP to site stakeholders (e.g., site employees, members of the public, scientists, regulators, environmental organizations) and gathering stakeholder input on the list of issues that should be addressed by the Program. Summaries of the discussions from these meetings as well as lists of the stakeholders who were invited to attend are included as Attachment 1.

In August/September 1996, The Keystone Center was asked to convene two additional site meetings using funds that remained in our contract. These meetings were held in October 1996 at Oak Ridge and Idahò National Engineering Laboratory. Summaries from these meetings and participant lists are included as Attachment 2.

In the Spring of 1997, Keystone staff met with EMSP staff to discuss additional opportunities for Keystone to work with the Program, using remaining funds. Keystone submitted a proposal outlining an assessment process and additional meetings if warranted by the information collected in the assessment. This proposal is included as Attachment 3. 

Following discussions on the proposed activities, Keystone initiated a site assessment process in August 1997. The goals of the assessment were to determine whether additional activities relating to the EMSP were desired and/or needed by the four sites where meetings had previously been held. The assessment consisted of Keystone staff conducting phone interviews and other research to answer these questions. The final report outlining the assessment findings is included as Attachment 4.

The Keystone Center's work under their original contract for this project was completed in October 1997.

fildata12691011016aaw.doc 

Following discussions on the proposed activities, Keystone initiated a site assessment process in August 1997. The goals of the assessment were to determine whether additional activities relating to the EMSP were desired and/or needed by the four sites where meetings had previously been held. The assessment consisted of Keystone staff conducting phone interviews and other research to answer these questions. The final report outlining the assessment findings is included as Attachment 4.

The Keystone Center's work under their original contract for this project was completed in October 1997.

f:Idata12691011016aaw.doc 


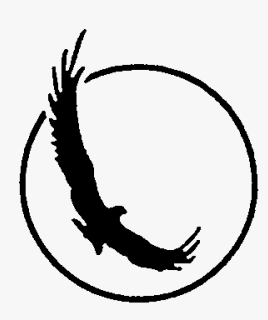

\section{The Keystone Center}

\section{Memorandum}

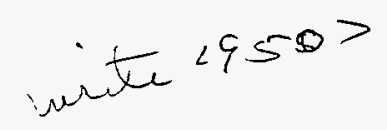

To: $\quad$ Jay Dodson, Program Acquisition and Assistance Division, U.S. Department of Energy

From: $\quad$ Kevin Curtis and Alyssa Wittenborn

Subject: $\quad$ Final Technical Report - Grant \# DE-FG03-96ER62220

Date: $\quad$ January 22, 1998

This memorandum shall serve as the Final Technical Report from The Keystone Center to the Department of Energy for our work with the Environmental Management Science Program (EMSP). This memorandum will describe briefly the various activities in which The Keystone Center engaged with the EMSP under Grant \# DE-FG03-96ER62220. The reports resulting from these activities, including four meeting summaries and a Final Report on Assessment Activities for EMSP, are attached for more detailed information.

The Keystone Center began its work with the EMSP in May, 1996, when The Center agreed to design, organize, and facilitate stakeholder meetings at two DOE sites: Savannah River and Hanford. These meetings were held in June, 1996 with the purposes of introducing the EMSP to site stakeholders (e.g., site employees, members of the public, scientists, regulators, environmental organizations) and gathering stakeholder input on the list of issues that should be addressed by the Program. Summaries of the discussions from these meetings as well as lists of the stakeholders who were invited to attend are included as Attachment 1.

In August/September 1996, The Keystone Center was asked to convene two additional site meetings using funds that remained in our contract. These meetings were held in October 1996 at Oak Ridge and Idaho National Engineering Laboratory. Summaries from these meetings and participant lists are included as Attachment 2.

In the Spring of 1997, Keystone staff met with EMSP staff to discuss additional opportunities for Keystone to work with the Program, using remaining funds. Keystone submitted a proposal outlining an assessment process and additional meetings if warranted by the information collected in the assessment. This proposal is included as Attachment 3. 
- Efficient Separation Processes

- Separation processes are needed to remove contaminants from waste streams without producing significant secondary wastes and to reduce the total volume of wastes generally.

- More effective and efficient processes are needed for removing trace amounts of contaminants from large volumes of water including ground water.

- Waste Treatment/Management

- More effective, less invasive treatment techniques such as bioremediation are needed.

- Information on the long term behavior of different waste disposal forms and containment media is needed to better inform decision-makers.

- Materials Stabilization

- Improved methods for predicting partitioning performance of vitrification techniques are needed.

B. Major Contaminants

Radionuclides were the foremost group of contaminants addressed by participants in terms of life cycle costs and general risk concerns.

- Radionuclides

- All aspects of Radionuclides need research. These tend to be a DOE-specific problem and are of tremendous concern to affected communities. TRU wastes, especially Pu 238, are particularly important to SRS which has approximately $30 \%$ of TRU wastes in weight and $80 \%$ in curies.

In addition, three other categories of contaminants, which would likely benefit from basic science research, were identified by participants.

- Organics

- There is particular concern with dense non-aqueous phase liquids (DNAPLs) and solvents in groundwater.

- Mixed and heterogeneous wastes

- The synergistic effects of mixed wastes need to be better understood.

- Heavy metals 


\section{Cross-Cutting Issues/Concerns}

Several other general areas of concern arose throughout the meeting. These issues cut across the conversations on basic research needs and warrant separate discussion.

\section{Criteria/Risk Assessment/Prioritization}

The group identified several criteria that they would like the EMSP to use in making decisions: health risks to the public, workers, and the environment; cost effectiveness; availability of treatment technologies; time sensitivity (i.e., regulatory requirements); and risks caused or exacerbated by remedial activities. These criteria were not prioritized by the group.

Issues specific to risk assessment included the need for better ways to communicate risks to the public and the need to develop a clear and systematic process for openly incorporating risk into cleanup priority setting.

Finally, concern was expressed that the costs associated with cleanup should not be the only determinate of which research to conduct. An example noted was the possibility of giving priority to known high life-cycle cost problem areas over problem areas that are potentially costly but presently without detailed cost information (e.g., focusing on high level waste treatment at the expense of groundwater remediation). Another example mentioned was the need to focus on problem areas that have created stakeholder concerns due to potential health risks.

\section{"Good Science" Concerns}

Several participants expressed concern over the need for the EMSP to avoid being overprescriptive in its research solicitations. They felt that the EMSP should fund basic science that is focused on problem areas while allowing researchers to be innovative and seek true "breakthroughs." Participants also stressed the need for educational and research support programs that would help encourage a next generation of scientists to focus specifically on DOE's contamination problems.

\section{Stakeholder Involvement}

Several participants raised questions concerning the appropriate role of public stakeholders who lack scientific and technical expertise. This discussion noted the current lack of communication between scientists and the public, and the public's subsequent lack of understanding of the problems and potential solutions. As one community representative pointed out, without understanding the technical aspects of the issues, particularly the associated risks, it is difficult for public stakeholders to have substantive input into the process and program.

Several stakeholders, representing public constituencies, provided DOE with written or verbal lists of concerns and questions concerning the EMSP and site cleanup generally. 


\section{Waste Management/Pollution Prevention}

One participant stated that he would like scientists to develop an "anti-waste generation pill" that would prevent the creation of new wastes during cleanup activities. There was discussion over whether this issue was a potential area for science research or simply a "cultural change" that must be accepted and incorporated by all cleanup decision makers.

\section{Communication Concerns}

Improved communication between all parties involved in and affected by cleanup issues was promoted throughout the meeting. Specifically, communication between researchers and environmental managers or problem holders must increase in order to promote mutual understanding and better link research to cleanup needs. Also, stakeholders voiced support for better communication between local stakeholders and DOE, both the site and headquarters. DOE needs to deliver consistent messages to stakeholders and improve the communication of important information to them. The communication of risks associated with contamination and cleanup on the site is particularly important.

\section{Next Steps}

- SRS stakeholders would like to see the EMSP prioritization criteria before it is used to make decisions.

- Stakeholders would like the EMSP to present its funding decisions for Fiscal Year 1996 to the site so stakeholders can see that their needs were heard and incorporated into the program.

- Participants expressed interest both in convening a group of basic science experts to identify general areas of needs and opportunities in science, and in further developing a site-specific basic science needs list or matrix.

- Several members of the group requested that the EMSP formally address the specific questions that were raised by stakeholders before and during the meeting.

- Savannah River stakeholders requested that DOE report to them the results of the Hanford EMSP meeting.

- Meeting participants expressed a general desire for a process to be developed that will help continue, expand, and improve stakeholder involvement in the science needs identification process and in the EMSP generally. 


\section{Environmental Management Science Program Workshop \\ June 24-25, 1996 \\ Savannah River, South Carolina}

\section{Invitation List}

* denotes confirmed participation

*Dr. Domy Adriano

Professor and Head

Biogeochemical Ecology

Savannah River Ecology Laboratory

University of Georgia

Drawer E

Aiken, SC 29801

(803) 7252472

Fax (803) 7253309

*Dottie Aiken

Business Manager

Education, Research and Dev. Assoc. of

Georgia Universities

900 Atlantic Drive

Atlanta, GA 30332-0425

(404) 8942087

Fax (404) 8949325

Mr. Charlie Anderson

U.S. Department of Energy

P.O. Box A

Aiken, SC 29802

(803) 2086084

Fax (803) 2086441

*Ms. Cynthia Anderson

Director, ERD

U.S. Department of Energy

P.O. Box A

Aiken, SC 29802

(803) 7253966

Fax (803) 7257548

*Dr. Stanley I. Auerbach

Senes, Oak Ridge

(Nat. AcadScience/Nat Research

Council/Committee on EMSP

103 Wildwood Drive

Oak Ridge, TN 37830

(423) 4835139

Fax (615) 4810060
*Connie Bailey

Waste Policy Institute

1872 Pratt Drive

Blacksburg, VA 24060

(540) 2319873

Fax (540) 2313968

Mr. Arthur Belge

Savannah River Citizens Advisory

Board

711 Coolbrook Court

Martinez, GA 30907

*Dr. Sally M. Benson

Division Director

Earth Sciences Division

E.O. Lawrence Berkeley Laboratory

1 Cyclotron Road

Building B90-1116

Berkeley, CA 94720

(510) 5865875

Fax (510) 4867714

Dr. Paul M. Bertsche

Professor

Biogeochemistry

Savannah River Ecology Laboratory-AACES

Savannah River Site

Drawer $\mathrm{E}$

Aiken, SC 29802

(803) 7255637

Fax (803) 7250077

Dr. Dennis Bickford

Westinghouse Savannah River

Company

Savannah River Site

Building 773-A

Aiken, SC 29808

Fax (803) 7254704 
*Ms. Allison Blackmon

Coordinator, SRS Site Technology

Coordinator Group

U.S. Department of Energy

P.O. Box A

Aiken, SC 29802

(803) 7259762

Fax.(803) 7253616

Mr. Charles Borup

U.S. Department of Energy

P.O. Box A

Aiken, SC 29802

(803) 7251425

Fax (803) 7255968

*Dr. Judith L. Bostock

Assistant Manager

Office of Science, Tech., \& Bus.

Development

U.S. Department of Energy-Savannah River Site

P.O. Box A

Aiken, SC 29802

(803) 7253821

Fax (803) 7255968

Ms. de'Lisa Bratcher

U.S. Department of Energy

P.O. Box A

Aiken, SC 29802

(803) 7255351

Fax (803) 7253616

*Don Bridges

U.S. Department of Energy

Dr. Michelle S. Broido Ph.D.

Acting Director

Environmental Sciences Division

Office of Health and Environmental

Research

U.S. Department of Energy

19901 Germantown Road

Germantown, MD 20874-1290

(301) 9033281

Fax (301) 9038519

Ms. Anne Brown

Savannah River Citizens Advisory

Board

275 Duncan Road

North Augusta, SC 29841
*Mr. James (Jim) P. Brown

Technical Advisor

Office of Science, Tech., \& Bus.

Development

U.S. Department of Energy

P.O. Box A

Aiken, SC 29802

(803) 7252760

Fax (803) 7252123

Dr. Michelle Buchanan

Oak Ridge National Laboratory

P.0. Box 2008

Oak Ridge, TN 37831

Fax (423) 574-0405

Mr. Scott Cannon

U.S. Department of Energy

P.O. Box A

Aiken, SC 29802

(803) 7252219

Fax (803) 7251440

*Dr. Walter E. Castro

Director of Special Projects

SC Universities Research \&

Education Foundation (SCUREF)

Strom Thurmond Institute

Clemson, SC 29634

(864) 6560954

Fax (864) 6560958

Mr. Emie Chaput

Deputy Manager

U.S. Department of Energy

P.O. Box A

Aiken, SC 29802

(803) 7253822

Fax (803) 7251910

Mr. Aundria D. Cheever

Savannah River Citizens Advisory

Board

360 Bay Street, Suite 435

Augusta, GA 30901

Dr. Gregory Choppin

Department of Chemistry

Florida State University

Tallahassee, FL 32306

Fax (904) 6443875 
Dr. Connie Cicero

Westinghouse Savannah River Company

Savannah River Site

Building 773-41A

Aiken, SC 29808

$\operatorname{Fax}(803) 7254553$

\section{Mr. Thomas W. Costikyan}

Savannah River Citizens Advisory

Board

6 Doe Point

Dataw, SC 30901

*Brian Costner

Director

Energy Research Foundation

537 Harden Street

Columbia, SC 29202

(803) 2567298

Fax (803) 2569116

*Dr. Todd V. Crawford

Consultant

1040 Woodland Drive

New Ellenton, SC 29809

(803) 6523856

Fax (803) 6523856

*Dr. David A. Dixon

Associate Director

Environmental Molecular Sciences

Laboratory

Pacific Northwest National Laboratory

Batelle Boulevard

P.O. Box 999

Richland, WA 99352

(509) 3724999

Fax (509) 3756631

Mr. Bill Donaldson

Savannah River Citizens Advisory

Board

204 Second Avenue

Tybee Island, GA 31328
*Ms. Martha E. Ebra

Level 3 Manager

Savannah River Technology Center

Westinghouse Savannah River Company

Savannah River Site

Building 773-A

Aiken, SC 29808

(803) 7253020

Fax (803) 7258136

*Mr. Donald L. Erich

Managing Director

Verification Research Laboratory

Clemson University

342 Computer Court

Anderson, SC 29625

(864) 6560792

Fax (864) 6560672

Elaine Faustmann

Consortium for Risk Evaluation \&

Stakeholder Participation (CRESP)

(206) 6852269

Fax (206) 6854696

*Dr. Mario Fiori

Manager

U.S. Department of Energy

P.O. Box A

Aiken, SC 29802

(803) 7253077

Fax (803) 7251910

Mr. Rick Ford

U.S. Department of Energy

P.O. Box A

Aiken, SC 29802

(803) 7252889

Fax (803) 7254657

*Dr. Nat Frazer

Associate Director

Savannah River Ecology Laboratory

University of Georgia

P.O. Drawer E

Aiken, SC 29802

(803) 7255728

Fax (803) 7253309 
*Perry Fulkerson

Director of Development for

Engineering \& Science

Administration \& Advanceme

Clemson University

110 Daniel Drive

Clemson, SC 29634

(864) 6560661

Fax (864) 6561692

Ms. Virginia Gardner

U.S. Department of Energy

P.O. Box A

Aiken, SC 29802

(803) 7259762

Fax (803) 7253616

Mr. Jim Gaver

Director

U.S. Department of Energy

P.O. Box A

Aiken, SC 29802

(803) 7252889

Fax (803) 7254657

*Mr. John Geiger

Deployment Manager

Subsurface Contaminants Focus Area

Office of Science, Tech., \& Bus.

Development

U.S. Department of Energy-Savannah River

Operations Office

P.O. Box A

Aiken, SC 29802

(803) 7251496

Fax (803) 7252123

Mr. Les Germany

U.S. Department of Energy

P.O. Box A

Aiken, SC 29802

(803) 7253966

Fax (803)-7257548
*Mr. Mark A Gilbertson

Director of Science \& Risk Policy

Office of Science \& Risk Policy

Technology Development

U.S. Department of Energy

Forrestal Building

1000 Independence Ave., SW

EM-52

Washington, DC 20585

(202) 5867150

Fax (202) 5861492

Mr. Stuart Goldstein

Special Assistant to Deputy Director

Office of Energy Research

U.S. Department of Energy

Forrestal Building

1000 Independence Ave., SW

ER-1

Washington, DC 20585

(202) 5860178

Fax (202) 5864120

*C.R. (Rudy) Goetzman

Westinghouse Savannah River Company

Building 999-W

Aiken, SC 29808

(803) 652-1845

Fax (803) 652-1898

Dr. Elizabeth Goodman

U.S. Department of Energy

P.O. Box A

Aiken, SC 29802

(803) 7253077

Fax (803) 7255968

Dr. Anthony Gouge

Westinghouse Savannah River Company

Savannah River Site

Building 779-2A

Aiken, SC 29808

Fax (803) 7254478

Mr. Allen Gunter

U.S. Department of Energy

P.O. Box A

Aiken, SC 29802

Fax (803) 9522019 
Mr. Tom Gutmann

U.S. Department of Energy

P.O. Box A

Aiken, SC 29802

(803) 2087408

Fax (803) 2086441

Dr. Dick Haire

Oak Ridge National Laboratory.

P.O. Box 2008

Oak Ridge, TN 37831

Fax (423) 5740405

*Mr. Edward A. Hamilton

Acting Chief Operating Officer

South Carolina Universities Research \&

Education Foundation

Strom Thurmond Institute

Clemson, SC 29634-0901

(864) 6560226

Fax (864) 6560958

*Ms. Dianna C. Hannah

Environmental Engineer

AMSTBD

U.S. Department of Energy

P.O. Box A

Aiken, SC 29802

(803) 7250403

Fax (803) 7255968

Dr. W. Franklin Harris

Director

Department of Biology, M-303

University of Tennessee

M383 Walters S4

Knoxville, TN 37922

(423) 9746841

Fax (423) 9744057

Ms. Dawn Haygood

Board Administrator

Savannah River Site

Building 730-2B, Room 1037

Aiken, SC 29808

(803) 9526971

Fax (803) 9526962
Dr. Terry Hazen

Westinghouse Savannah River Company

Savannah River Site

Building 773-42A

Aiken, SC 29808

Fax (803) 7258136

*Mr. Tom Heenan

Assistant Manager

U.S. Department of Energy

P.O. Box A

Aiken, SC 29802

(803) 7258074

Fax (803) 7253616

*Dr. Carol J. Henry

Associate Deputy Assistant Secretary

Office of Science \& Technology

Office of Environmental Management

U.S. Department of Energy

Forrestal Building

1000 Independence Avenue, SW

EM-50

Washington, DC 20585

(202) 5867150

Fax (202) 5861492

Mr. Tom Hicks

U.S. Department of Energy

P.O. Box A

Aiken, SC 29802

(803) 7252760

Fax (803) 7252123

*Tom Hinton

Assistant Research Ecologist

University of Georgia

Savannah River Ecology

Laboratory

Drawer E

Aiken, SC 29802

(803) 5577454

Fax (803) 5577324 
*Dr. Roland Hirsch

Acting Director

Medical Applications \& Biophysical

Research Division

Office of Health \& Environmental

Research

Office of Energy Research

U.S. Department of Energy

19901 Germantown Road

Germantown, MD 20874-1290

(301) 9033213

Fax (301) 9030567

Mr. Albert M. Hodge

President

Metro Augusta Chamber of Commerce

P.0. Box 657

Augusta, GA 30903-0657

(706) 8211300

Fax (706) 8210657

*Dr. Karen L. Hooker

Director

Program Management \& Coordination

U.S. Department of Energy

Office of Environmental Quality

P.O. Box A

Aiken, SC 29802

(803) 7259615

Fax (803) 7253616

Dr. Paul G. Huray

Carolina Distinguished Professor

South east Manufacturing Technology Center

Swearingen Engineering Center

University of South Carolina

Columbia, SC 29208

(803) 777-9520

Fax (803) 777-9557

*Dr. Pat Jackson

U.S. Department of Energy

P.O. Box A

Aiken, SC 29802

(803) 7251425

Fax (803) 7255968
*Morris James

Stakeholder/Regulatory Coordinator

Office of Science, Tech., \& Bus.

Development

U.S. Department of Energy-

Savannah River

P.O. Box A

Aiken, SC 29802

(803) 7255960

Fax (803) 7255968

Dr. Carol Jantzen

Westinghouse Savannah River Company

Savannah River Site

Building 773-A

Aiken, SC 29808

Fax (803) 7254704

*Brendolyn L. Jenkins

Savannah River Citizens Advisory

Board

P.O. Box 228-Main Street

Elko, SC 29826

(803) 2665454

Fax (803) 2665787

*Mr. Brad Jenkins

Robotics \& Remote Systems

Savannah River Technology Center

Westinghouse Savannah River

Company

Building 773-A, Room D-1132

Aiken, SC 29808

(803) 7258764

Fax (803) 7257369

*Ms. Pam Jenkins

LLW Program Manager

Solid Waste

U.S. Department of Energy-SR

P.0. Box A

Aiken, SC 29802

(803) 7251541

Fax (803) 7251440

*Ms. Gail Jernigan

Environmental Scientist

Westinghouse Savannah River Company

Building 773-41A

Aiken, SC 29808

(803) 7254535

Fax (803) 7252978 
Mr. Thomas Johnson

U.S. Department of Energy

P.O. Box A

Aiken, SC 29802

(803) 7253966

Fax (803) 7257548

Mr. Thelonious A. Jones

Savannah River Citizens Advisory .

Board

2010 Lanier Road

Augusta, GA 30909

Dr. Ratib Karam

Director

Georgia Institute of Technology

Education, Research and Development

Association

900 Atlantic Drive

Atlanta, GA 30322-0425

(404) 8943600

Fax (404) 8949325

*Mr. Will LaVeille

Technical Program Officer

Program Management \& Coordination Division

U.S. Department of Energy-SRS

P.O. Box A

Aiken, SC 29802

(803) 7257663

Fax (803) 7253616

Mr. William F. Lawless

Co-chair

ERWM Subcommittee

Paine College

Mathematics Department

1235 15th Street

Augusta, GA 30901-3182

7068218335

Fax 7068218396

Dr. W. Ivan Lewis

Westinghouse Savannah River

Company

Savannah River Site

Building 773-A

Aiken, SC 29808

Fax (803) 7257369
Ms. Ann G. Loadholt

Savannah River Citizens Advisory .

Board

Dr. Brian Looney

Westinghouse Savannah River

Company

Savannah River Site

Building 773-41A

Aiken, SC 29808

Fax (803) 725-8136

*Karen Lowrie

Consortium for Risk Evaluation \&

Stakeholder Participation (CRESP)

681 Frelinghaysen Road

Piscataway, NJ 08855

(908) 4450521

Fax (908) 4450959

*William C. Luth

Division Director

Division of Engineering \& Geosciences

U.S. Department of Energy

19901 Germantown Road

Germantown, MD 20874

(301) 9035822

Fax (301) 9030271

*Ms. Cindy Malencia

Stakeholder Coordinator

Subsurface Contaminants Focus Area

U.S. Department of Energy

P.O. Box A

Aiken, SC 29802

(803) 7252123

Fax (803) 7252123

Ms. Jennifer Marek

U.S. Department of Energy

P.O. Box A

Aiken, SC 29802

(803) 9523107

Fax (803) 9522019

*Ms. Ramoncita Massey

U.S. Department of Energy

19901 Germantown Road

EM-62

Germantown, MD 20874-1290

(301) 9035059

Fax (301) 5867192 
Ms. Suzanne Matthews

Savannah River Citizens Advisory Board 152 Dupree Place

Aiken, SC 29801

Ms. Kathryn May

Co-chair

ERWM Subcommittee

U.S. Department of Energy

3597 Pebble Beach Drive

Martinez, GA 30907

(706) 8603249

*Dr. Mildred McClain

Citizens for Environmental Justice

720 Maupas Avenue

Savannah, GA 31401

(912) 2330907

Fax (912) 2335105

Mr. Larry McKinney

Savannah River Citizens Advisory

Board

*Mr. Scott McMullin

Containment Deployment Sector

Manager

Subsurface Contaminant Focus Area

U.S. Department of Energy

P.O. Box A

Aiken, SC 29802

(803) 725-9596

Fax (803) 7252123

Ms. Jo-Ann Nestor

Savannah River Citizens Advisory

Board

P.O. Box 6915

Hilton Head Island, SC 29938

${ }^{*}$ Mr. William L. Noll

Division Director

Solid Waste Division

U.S. Department of Energy-Savannah River

Operations Office

P.O. Box A

Aiken, SC 29802

(803) 7252219

Fax (803) 7251440
Mr. Dan Oakley

Senior Scientist

Washington Policy Institute

555 Quince Orchard Road

Suite 600

Gaithersburg, MD 20878

Fax (301) 9906150

*Mr. Dale Ormand

Senior TRU Waste Program Manager-SRS-

Solid Waste

Division

U.S. Department of Energy

P.O. Box A

Aiken, SC 29802

(803) 7258013

Fax (803) 7251440

Dr. Anthony V. Palumbo

Oak Ridge National Laboratory

P.O. Box 2008

Oak Ridge, TN 37831

Fax (423) 5740405

*Dr. Lucien M. Papouchado

Manager, Waste \& Environmental

Technology

Savannah River Technology

Center

Westinghouse Savannah River

Company

Building 773-A

Aiken, SC 29808

(803) 7253701

Fax (803) 7251660

*Mr. Lane D. Parker

HaxMat Director

IUOE Local 470

P.O. Box 2462

Aiken, SC 29802

(803) 6480729

Fax (803) 6480720

Ms. Karen Patterson

Savannah River Citizens Advisory

Board

1103 Conger Drive

Aiken, SC 29803

(803) 2796363

Fax (803) 2799523 
*W . Lee Poe Jr.

Citizen Stakeholder

807 E. Rollingwood Road

Aiken, SC 29801

(803) 6427297

Fax (803) 6427297

*Mr. Jim Pope

U.S. Department of Energy

Mr. Howard Pope

U.S. Department of Energy

P.O. Box A

Aiken, SC 29802

(803) 7252219

Fax (803) 7251440

Mr. Phil Prater

U.S. Department of Energy

P.O. Box A

Aiken, SC 29802

(803) 7253966

Fax (803) 7257548

Ms. Ann Ragan

Federal Facility Liaison

SCDHEC, EQC-ADM

2600 Bull Street

Columbia, SC 29201

(803) 7344721

Fax (803) 7349196

*Dr. Kamalakar R. Raut Ph.D.

Savannah River Citizens Advisory

Board

Savannah State University

708 Penn Waller Road

Savannah, GA 31410

(912) 8971427

Fax (912) 8971637

Mr. Andrew W. Rea

Citizens for Clean Air and Water

55 E. Deerwood Road, Apt. 17

Savannah, GA 31410

*Kathie Reed

U.S. Department of Energy

Forrestal Building

1000 Independence Ave., SW

Washington, DC 20585
Mr. Dennis Ryan

U.S. Department of Energy

P.O. Box A

Aiken, SC 29802

(803) 7253077

Fax (803) 7255968

*Dr. Gary S. Sayler

Professor \& Director

Center for Environmental Biotechnology

University of Tennessee

10515 Research Drive, Suite 100

Knoxville, TN 37932

(423) 9748080

Fax (423) 9748086

*George Schneider

(208) 5266789/Fax (208) 5266249

*Ms. Margaret G. Schwenker

SRTC Program Manager

Assistant Manager Office for

Science, Tech., \& Bus.

Development

U.S. Department of Energy-SR

P.O. Box A

Aiken, SC 29802

(803) 7250403

Fax (803) 7255968

Ms. Deborah Simone

Savannah River Citizens Advisory

Board

5751 Begonia Lane

Thomson, GA 30824

*Mr. Len Sjostrom

Assistant Manager

U.S. Department of Energy

P.O. Box A

Aiken, SC 29802

(803) 7255562

Fax (803) 7258272

Mr. Robert H. (Bob) Slay

Board Chair

Savannah River Citizens Advisory

Board

P.O. Box 192

Beech Island, SC 29842

(803) 3021210

Fax (803) 3021210 
*Dr. Rich Smalley

Senior Environmental Scientist

Waste Policy Institute

22 Gateway Drive

Aiken, SC 29803

(803) 6528020

Fax (803) 6528484

*Perjetta Smith

Savannah River Citizens Advisory

Board

Fax (803) 5579642

*Dr. Michael H. Smith

Director

Savannah River Ecology

Laboratory

University of Georgia

Drawer E

Aiken, SC 29802

(803) 7252959

Fax (803) 7253309

Dr. Leonard Spicer

Duke University Medical Center

Department of Biology

Box 3711

235 Nanaline Duke

Durham, NC 27710

(919) 6848111

Fax (919) 6848885

*William Ed Stevens

Manager, Solid Waste \& Special

Programs

Interim Waste Technology Section

Savannah River Technology Center

Westinghouse Savannah River Company

P.O. Box 616

Aiken, SC 29802

(803) 725-7751

Fax (803) 725-4704

*Dr. Carl Strojan

Research Manager

Savannah River Ecology Laboratory

University of Georgia

P.O. Drawer E

Aiken, SC 29803

(803) 7258217

Fax (803) 7253309
*Mr. Harold F. Sturm Jr.

Manager, Environmental

Restoration Technology

Savannah River Technology

Center

Westinghouse Savannah River

Company

Savannah River Site

Building 773-A, Room A-233

Aiken, SC 29808

(803) 7253497

Fax (803) 7258136

Mr. J. Ed Tant

Savannah River Citizens Advisory

Board

5047 Victoria Avenue

North Charleston, SC 29405

*Dr. Tom Temples

U.S. Department of Energy

P.O. Box A

Aiken, SC 29802

(803) 7253966

Fax (803) 7257548

*Mr. Brian J. Teppen

Assistant Research Professor

Biogeochemistry

Savannah River Ecology

Laboratory-AACES

Drawer E

Aiken, SC 29802

(803) 7258157

Fax (803) 7250077

Mr. Eric P. Thompson

Executive Director

Lower Savannah Council of

Governments

P.O. Box 850

Aiken, SC 29802

(803) 6497981

Fax (803) 6492248

Mr. Tom Treger

U.S. Department of Energy

P.0. Box A

Aiken, SC 29802

(803) 7253966

Fax (803) 7257548 
Ms. Camilla Warren

Chief Remedial Section

U.S. Environmental Protection Agency

1345 Courtland Street, NE

Atlanta, GA 30365

(404) 3473016

Fax (404) 3475205

*Mr. Lee Watkins

U.S. Department of Energy

P.O. Box A

Aiken, SC 29802

(803) 2086053

Fax (803) 2086441

*Ms. Audrey B. Watson

Community Leader Network

Environmental Education

The Garden Club of South Carolina

910 River Oak Drive

North Augusta, SC 29841

(803) 2798420/Fax (803) 2790833
Ms. Beaurine $\mathrm{H}$. Wilkins

Savannah River Citizens Advisory

Board

2524 Rhodes Drive

Augusta, GA 30906

*Dr. Susan Wood

Vice President and Director

Westinghouse Savannah River

Company

Savannah River Site

Building 773-A

Aiken, SC 29808

Fax (803) 7258136

Mr. Vernon Zinnerman

Savannah River Citizens Advisory

Board

P.O Box 181

Augusta, GA 30903 
$\sin x$ 
2 
2 


\section{Summary of the Environmental Management Science Program Workshop June 27-28, 1996}

\section{Richland, WA}

\section{Presentations and Opening Remarks}

On June 27-28, 1996, the Department of Energy (DOE) hosted a meeting to discuss its Environmental Management Science Program (EMSP) and the program's role in constructing a site-specific basic research agenda that maps site cleanup needs to basic science areas. Please see the attached list of those who were invited to the workshop.

The meeting opened with an introduction by Robert Rosselli, Assistant Manager, Science and Technology, Richland Operations Office. Drs. Carol Henry and Roland Hirsch followed with presentations on Environmental Management and Energy Research respectively, and on the joint Environmental Management Science Program. From the perspective of the EMSP, the stated objectives for the meeting were to inform the site and its stakeholder community about the EMSP including its history, purpose, progress, and goals; and to initiate a dialogue with the Hanford Nuclear Site (Hanford) and its stakeholders on developing a research agenda that maps basic science research to site-specific research needs. This agenda, when developed, will be used to help inform and guide the national EMSP program as well as assist the site.

Mr. John Wagoner, Site Manager, then gave a presentation detailing the site's cleanup plan and opportunities for science to contribute to the cleanup effort. The final presentation on Thursday was delivered by Dr. Teresa Fryberger, Deputy Director, Environmental Molecular Sciences Laboratory, on the ways in which basic science can contribute to cleanup. Debbie Trader of Hanford also gave a brief and informal presentation on the Hanford Site Technology Coordination Group.

After the presentations, the participants of the workshop engaged in discussions regarding:

- the Fiscal Year 1996 EMSP proposal selection process, including a list of major cleanup problems that exist at Hanford which could benefit from basic science research efforts;

- the EMSP beyond Fiscal Year 1996, including aspects of the decision-making process, and identified areas for greater communication and collaboration among researchers and users; and

- development of a Hanford-specific basic science research agenda.

These discussions were not intended to result in a consensus among the participants regarding any of the advice given or points raised during the meeting. Further, this summary is not a consensus document. Rather, the points made in this summary were offered by workshop participants who were speaking as individuals, and not formal representatives of their agencies or organizations. This summary tries to capture the major points made during the meeting and is organized around the points above. It does not attribute comments to any participant, and does not follow the same chronology of the points raised in the discussion. 


\section{The Fiscal Year 1996 EMSP Proposal Selection Process}

\section{General Feedback:}

Several participants indicated that it was difficult to provide advice given the short time frame of the EMSP proposal process for Fiscal Year 1996, but that the EMSP could provide a catalytic effect for better integrating basic science research with cleanup needs. It also was requested by several participants that those involved in making the selections for Fiscal Year 1996 communicate the criteria used for the decision making to interested stakeholders so that they can provide more input in the future.

Workshop participants were generally supportive of efforts to better link basic science research carried out by the DOE's Energy Research program to site-specific cleanup needs. Participants also generally endorsed the statement that basic science can meet needs throughout the spectrum of technology maturation.

\section{Identified Problem Areas:}

Workshop participants were asked to identify problem areas where basic science research could assist cleanup in the future. In addition, several lists of needs, problems, and cleanup priorities generated by other workgroups at Hanford prior to the meeting were distributed. A list of these documents is attached for reference, but it should be noted that they were not endorsed by workshop participants and are not products of the workshop. In addition, several presentations went into greater detail to identify areas which could benefit from basic research. In particular, please see Mr. Wagoner's and Dr. Fryberger's presentations.

During the workshop, a distinction was made between environmental problems, such as contamination in groundwater that exist at the site, and the needs that the site must fulfill to address those problems, such as developing new groundwater treatment technologies. It was acknowledged that basic science research can both address problems and help fulfill needs. However, it was suggested that there should be a focus on identifying the problems because, while the needs may change over the next ten years as technologies mature, the problems where basic science research may have the greatest potential impact, will endure.

The following were identified by participants as problem areas that could benefit from basic science research:

- Characterization of contamination in the soil and groundwater, including understanding mobility of contaminants in the subsurface;

- Removal/remediation of contaminants in groundwater and soils (e.g. dense non-aqueous phase liquids (DNAPLs) in groundwater), including subsurface transport of contaminants, bioremediation, and long-term health effects of remaining contaminants; 
- Tank waste remediation, including characterization, safety, tank processing, and waste forms;

- Identification of populations at risk, including risks to workers and to the ecological systems on-site, such as the toxicological risks associated with mixed radiological and chemical wastes;

- Definition of the real risk to those populations;

Several participants discussed the shortcomings of risk assessments, and how they are used. It was noted that risk assessments should:

-- be credible

-- be consistent

-- be based on science

-- involve stakeholders

-- use the same pool of data

-- be clear about the assumptions being used

However, participants agreed that of the above factors, only one dealt with science, and should be discussed in other forums.

- Reduction of generation of wastes produced by remediation;

- "Breakthrough" concepts to facilitate cost reductions;

- The ultimate disposition of wastes including cesium capsules, plutonium, and reactors; -Several workshop participants expressed concern regarding a contingency plan if the Waste Isolation Pilot Plant (WIPP) and/or Yucca Mountain facilities are not available to accept Hanford wastes. It was suggested that efforts should be made to determine what the disposition of these wastes will be in the event that these facilities do not open. These efforts could include research that looks into developing stable waste forms that could be stored on-site.

- Nuclear materials "breakthroughs," including understanding the behavior of long-lived radionuclides in the environment;

- In-situ techniques for 200 Area waste deposits;

- K-Basin water clarity;

- Control of radioactive emissions, especially from waste processing activities; and

- Availability of adequate funding for additional research into the possible impact of seismic activity on the Hanford site. 


\section{EMSP beyond Fiscal Year 1996}

\section{General Feedback:}

Workshop participants spent a major portion of the workshop discussing how to improve communication among researchers and users or "problem holders." The main points from those discussions are summarized below under "Establishing a Collaborative Process." There were several general points made about the EMSP, however, that are listed immediately below.

- There is a need to develop a cadre of both basic and applied scientists who can better meet cleanup needs throughout the technology maturation process.

- The EMSP also has the potential to address the cleanup needs of several countries around the world and that a longer term goal of the EMSP could be to make this technology and expertise available to other countries. It was noted that this may be a way to recoup some of the expense of the EMSP and the entire DOE cleanup effort.

- In order to truly link basic research with cleanup needs, the program may have to be broader than the grant proposal process that currently makes up the EMSP. Several different models were suggested (see "Establishing a Collaborative Process").

- In the future, in order to create truly collaborative proposals, submitters need more time to prepare the proposals.

- The Environmental Molecular Sciences Laboratory should also be better integrated with cleanup priorities. It was noted however, that there are restrictions due to funding sources that affect how this integration can be achieved.

- The EMSP should be held accountable, in that there should be some "return on investment" criteria used in measuring basic research.

- Funding for research programs and for Environmental Management needs to be stable in order to attract high quality scientists and science.

\section{Establishing a Collaborative Process:}

Another major portion of the workshop discussion focused on establishing a process to allow for more communication and collaboration among basic researchers and those implementing the technologies that will benefit from basic research. Overall, it was generally agreed that two way communication between the research and scientific community and the users/problem holders is essential. It was noted that both groups, while pursuing different objectives, can better understand one another and the possible benefits to cleanup that may arise from basic research. Specifically the following general "principles" were offered to help guide future collaboration and communication.

- Decisions should be made on the merits of science, not politics.

- Researchers need to better understand cleanup problems. However, limiting research to explore areas only where there is a clear need (i.e., "streetlight" science) will not solve all problems. 
- The "just do it" focus of program managers, regulators, and other stakeholders should better incorporate new knowledge and information that results from basic science research.

- Investing in basic research should be viewed as investing in the reduction of uncertainties involved in cleanup decisions. This will allow for more cost-effective engineering.

- Programmatic decisions could benefit from better scientific information.

More specifically, the following points were made regarding the type of information that should be exchanged, and in what forum.

- Science needs should be identified and communicated to researchers early in the cleanup decision-making process. Communication of needs should continue to occur frequently throughout the spectrum of technology maturation. Examples included the various sites hosting meetings to which scientists are invited, and the converse in which a National Laboratory would invite program managers to visit the lab in order to better understand the types of science research underway.

- Researchers need to communicate the potential implications of different cleanup decisions to decision-makers before action is taken. Specifically, decision-makers need to be informed when certain cleanup decisions they are making could foreclose future options for cleanup.

- There are several different models for sharing information and enhancing communication which could be used, including fellowships, teams that include both researchers and users, and other models.

- Different funding mechanisms (other than the grant proposal process) may be needed to encourage a true team effort that will effectively feed into the cleanup decision-making process.

\section{Establishing a Site-Specific Basic Science Research Agenda for Hanford}

The importance of establishing a Hanford-specific basic science research agenda was discussed by several participants, both from the site and from the EMSP.

Many participants noted that in order to develop a research agenda for Hanford, it needs to be based on the long-term cleanup goals for the site, which in turn, need to be based upon a longterm vision for the site. It was suggested that the Hanford Strategic Plan may be an appropriate starting point for identifying these goals.

In addition, it was pointed out that developing such an agenda will require the input of projectlevel managers to adequately identify the needs and decision points. It will then be necessary to communicate how those needs will be met through EM-50 and other DOE programs.

It was also suggested that there be better communication between DOE headquarters and the site in order to reduce revisiting work and failure. It was added however, that ongoing evaluation may result in a genuine need to revisit work that has already been performed. 


\section{Next Steps}

The following next steps were identified at the workshop.

1. The EMSP managers need to communicate the decisions made for EMSP in Fiscal Year 1996, and the criteria used to make those decision, to all interested stakeholders.

2. The Site Technology Coordination Group (STCG) will assume responsibility to continue the dialogue of how to better link basic research to Hanford's cleanup needs. They will broaden participation as necessary to include the research and scientific community and program managers. The role of the Hanford Advisory Board (HAB) in this dialogue will be addressed through discussions with the HAB and the STCG.

3. The STCG also will coordinate efforts to provide input for the next EMSP solicitation regarding Hanford's needs.

f:Idatal269108lhanford2.doc 


\section{Environmental Management Science Program Workshop \\ June 27-28, 1996 \\ Richland, Washington}

Invitation List

*denotes confirmed participation

Mr. Jacob Bahren

Oak Ridge National Laboratories

P.0. Box 2008

Oak Ridge, TN 37831-6355

(423) 5747131

Fax (423) 5740405

*Mr. Gary Ballew

Site Technology Coordinator

Environmental Technology Partnership

Washington Department of Community,

Trade and Economic Development

1314 West 4th Avenue

Kennewick, WA 99336

(509) 7365711

Fax (509) 7363030

Dr. Scott Barnhart M.D., MPh

Director of Occupation \& Environmental

Medicine

Harborview Medical Center

325 9th Avenue

MS-359739

Seattle, WA 98104-2499

(206) 7313388

Fax (206) 7318247

Mr. Paul Beaver

U.S. Environmental Protection Agency

712 Swift Boulevard, Suite 5 -

MSIN B5-01

Richland, WA 99352

(509) 3768665

Fax (509) 3762396
Mr. Dick Belsey

Physicians for Social Responsibility

030 SW Ridge Drive

Portland, OR 97219-6566

(503) 2930225

Fax (503) 2930225

Ms. Rose Bennett

Nuclear Emergency Coordinator

Oregon Department of Energy

625 Marion Street, NE

Salem, OR 97310

(503) 3737400

Fax (503) 3786457

*Mr. James D. Berger

Manager

Office of Technology Integration

Westinghouse Hanford Company

Environmental Restoration-Waste

Management

LO-18, P.O. Box 1970

Richland, WA 99352

(509) 3769942

Fax (509) 3769746

Dr. Michelle S. Broido Ph.D.

Acting Director

Environmental Sciences Division

Office of Health and Environmental

Research

U.S. Department of Energy

19901 Germantown Road

Germantown, MD 20874-1290

(301) 9033281

Fax (301) 9038519 
${ }^{*}$ Mr. Chuck Broscious

Executive Director

Environmental Defense Institute

P.O. Box 220

Troy, ID 83871

(208) 8356152

Fax (208) 8356152

*Robert L. Brown

U.S. Department of Energy-Idaho

Operations Office

850 Energy

Idaho Falls, ID 83401

(208) 5265289

Fax (208) 5265964

*Ms. Pam Brown

Hanford Analyst

Hanford Communities

P.O. Box 190

505 Swift Boulevard

Richland, WA 99352

(509) 9437348

Fax (509) 9435666

Mr. Dennis Brown

U.S. Department of Energy

Richland Operations Office

P.O. Box 550, MSIN A5-52

Federal Building, 825 Jadwin Avenue

Richland, WA 99352

(509) 3769210

Fax (509) 3766621

*Dr. Larry C. Brown

Manager, Engineering Testing \&

Technology Projects

Projects \& Site Services

Westinghouse Hanford Company

P.O. Box 1970

MSIN L5-62

Richland, WA 99352

(509) 3762579

Fax (509) 3769964

Gordon Brown

GeoSciences Division

Stanford University

Palo Alto, CA

Fax (415) 7252199
Mr. Tom Carpenter

Government Accountability Project

West Coast Office

1402 Third Avenue, Suite 1215

Seattle, WA 98101

(206) 2922850

Fax (206) 2920610

Mr. Kevin V. Clarke

U.S. Department of Energy

Richland Operations Office

P.O. Box 550, A7-75

Federal Building, 825 Jadwin Avenue

Richland, WA 99352

(509) 3766332

Fax (509) 3760306

Dr. James A. Cochran

Campous Dean

Washington State University at Tri-Cities

100 Sprout Road

Richland, WA 99352

(509) 3727258

Fax (509) 3727354

Ms. Lynn Coleman

Washington State Department of

Ecology

Toxics Clean Up Program

300 Desmond Drive, SE

Lacey, WA 98503

(360) 4077194

Fax (360) 4077154

Mr. Roger Collis

Washington Environmental Technology

Partnership

2001 6th Avenue

Suite 2700

Seattle, WA 98121

(206) 4646282

Fax (206) 4645868

*Mr. Steven D. Colson

Associate Director, Chemical Structure

\& Dynamics

Pacific Northwest National Laboratories

MS K2-14, P.O. Box 999

Battelle Blvd.

Richland, WA 99352

(509) 3756882

Fax (509) 3726086 
*Mr. Robert Cook

Technical Analyst

Yakima Indian Nation

1933 Jadwin Avenue, \#110

Richland, WA 99352

(509) 9460101

Fax (509) 9438555

Mr. Kevin Crowley

National Academy of Sciences

National Research Council

2001 Wisconsin Avenue, NW

Washington, DC 20007

(202) 3343066

Fax (202) 3343077

Mr. Rico Cruz

Nez Perce Indian Nation

Main Street

Beaver Grade, ID 83540

(208) 8437375

Fax (208) 8437378

Mr. Greg de Bruler

Columbia River United

P.O. Box 667

Bingen, WA 98605

(509) 4932808

Fax (509) 4932808

Mr. David Dillman

Senior Vice President

TRIDEC

901 N Colorado

Kennewick, WA 99336

(509) 7351000

Fax (509) 7356609

*Dr. David A. Dixon

Associate Director

Environmental Molecular Sciences

Laboratory

Pacific Northwest National Laboratory

Batelle Boulevard

P.O. Box 999

Richland, WA 99352

(509) 3724999

Fax (509) 3756631
*Stephen Domotor

U.S. Department of Energy

19901 Germantown Road .

EM-52/Trevion II Room \#440 234/TRE

Germantown, MD 20874-1290

(301) 9035053

Fax (301) 9039770

Dr. Thom Dunning

Pacific Northwest National Laboratories

MS K2-20

Battelle Boulevard

Richland, WA 99352

(509) 3756863

Fax (509) 3756916

Dirk Dunning

Oregon Department of Energy

625 Marion Street, NE

Salem, OR 97310

(503) 785489

Fax (503) 3737806

Mr. Tom Engle

Professor

University of Washington

Department of Chemistry

Box 351700

Seattle, WA 98195-1700

(206) 6852330

Fax (206) 6858665

*Mr. John L. Erickson

Head of Environmental Radiation

Section

Division of Radiation Protection

Washington State Department of Health

P.0. Box 47827

Olympia, WA 98504-7827

(360) 5863306

Fax (360) 7531496

*Mr. Dennis Faulk

Remedial Project Manager

Office of Environmental Cleanup

U.S. Environmental Protection Agency

712 Switt Boulevard

Suite 5

Richland, WA 99352

(509) 3768631

Fax (509) 3762396 
Mr. Martin Fleck

Washington Physicians for Social

Responsibility

4554 12th Avenue, NE

Seattle, WA 98105

(206) 5472630

Fax (206) 5472631

*Mr. Chuck Foreman

(509) 372-3767

Mr. Clyde Frank

Deputy Assistant Secretary

U.S. Department of Energy

Technology Development

Forrestal Building

1000 Independence Avenue, SW

5B-014

Washington, DC 20585

(202) 5866382

Fax (202) 5866773

*Dr. Teresa Fryberger

Deputy Director

Environmental Molecular Sciences Lab

Pacific Northwest National Laboratories

P.O. Box 999, MS K2-20

Batelle Blvd.

Richland, WA 99352

(509) 752709

Fax (509) 3756916

*Mr. Ron Gerton

DOE-RL

U.S. Department of Energy

Richland Operations Office

P.O. Box 550, MSIN S7-50

Federal Building, 825 Jadwin Avenue

Richland, WA 99352

(509) 3766550

Fax (509) 3762002

*Mr. Stuart Goldstein

Special Assistant to Deputy Director

Office of Energy Research

U.S. Department of Energy

Forrestal Building

1000 Independence Avenue, SW

ER-1

Washington, DC 20585

(202) 5860178

Fax (202) 5864120
*Mr. Rick Gonzalez

U.S. Department of Energy

Richland Operations Office

P.0. Box 550, MSIN R3-79

825 Jadwin Avenue

Richland, WA 99352

(509) 3739922

Fax (509) 3760695

*Dr . Dibakar (Dib) Goswami

Senior Hydrogeologist

Washington Department of Ecology

Nuclear Waste Program

1315 W. 4th Avenue

Kennewick, WA 99336-6018

(509) 7363015

Fax (509) 7363030

Michael W. Grainey

Assistant to the Director

Oregon Department of Energy

625 Marion Street, NE

Salem, OR 97310

(503) 3785489

Fax (503) 3737806

Mr.Charles Hansen

U.S. Department of Energy-Richland Operations

Office

Waste Management

P.O. Box S7-41

Richland, WA 99352

(509) 3767434

Fax (509) 3730205

*Mr. Mark S. Hanson

Manager, Environmental Management Programs

Environmental Technology Division

Pacific Northwest National Laboratories

P.O. Box 999

3200 Q Avenue

Richland, WA 99352

(509) 3756812

Fax (509) 3754422

Mr. Stuart Harris

Confederate Tribes of the Umatilla Indian

Reservation

P.0. Box 638

Pendleton, OR 97801

(503) 2785211

Fax (503) 276-3095 
*Mr. Geoffrey L. Harvey

Public Information Specialist

Pacific Northwest National Laboratories

P.O. Box 999, KG-51

Battelle Boulevard

Richland, WA 99352

(509) 3739931

$\operatorname{Fax}(509) 3730628$

Mr. Harold Heacock

Hanford Advisory Board

760 S. Takoma

Kennewick, WA 99336

(509) 5864960

Fax (509) 5868289

*Ms. June M. Hennig

(509) 3724025

*Dr. Carol J. Henry

Associate Deputy Assistant Secretary

Office of Science \& Technology

Office of Environmental Management

U.S. Department of Energy

Forrestal Building

1000 Independence Avenue, SW

EM-50

Washington, DC 20585

(202) 5867150

Fax (202) 5861492

*Mr. Mark Hermanson

Westinghouse Humford Company

MISN 51-52

P.O. Box 1970

Richland, WA 99352

(509) 3762257

Fax (509) 3735635

*Dr. Roland Hirsch

Acting Director

Medical Applications \& Biophýsical

Research Division

Office of Health \& Environmental

Research

Office of Energy Research

U.S. Department of Energy

19901 Germantown Road

Germantown, MD 20874-1290

(301) 9033213

Fax (301) 9030567
Dr. Robert J. Huggett

Assistant Administrator

U.S. Environmental Protection Agency

Office of Research \& Development

401 M Street, SW

MC-8101

Washington, DC 20460

(202) 2607676

Fax (202) 2609761

*Ms. Jennie Hunter-Cevera

Head, Environmental

Biotechnology Earth

Lawrence Berkeley National Laboratory

Center for Environmental Biotechnology

Building 70A, MS 3317

1 Cyclotron Road

Berkeley, CA 94720

(510) 4867359

Fax (510) 4867152

*Mr. Ron Izatt

Deputy Manager

Environmental Management

U.S. Department of Energy-Richland Operations

Office

P.O. Box 550

MSIN A7-50

Richland, WA 99352

(509) 3764216

Fax (509) 3764789

*Mr. Russell Jim

Manager

Yakima Indian Nation

Environmental Restoration/Waste Management

Program

P.O. Box 151

2808 Main Street

Toppenish, WA 98903

(509) 4522502

Fax (509) 4522503

*Dr. Robert J. Julian

Environmental Specialist

Washington State Department of

Ecology

1315 West 4th Street

Kennewick, WA 99336

(509) 7365702

Fax (509) 7363030 
Ms. Judith Jurji

Hanford Downwinders Coalition

916 N. 36th Street

Seattle, WA 98103

(206) 5471021

Fax (206) 5475971

Professor Ronald Kathryn

Washington State University

100 Sprout Road

Richland, WA 99352

(509) 3727288

Fax (509) 3751817

Mr. Jim Kautzky

Strategic Planning Organizer

U.S. Department of Energy-Richland Operations

Office

P.O. Box 550, MSIN A5-58

Richland, WA 99352

(509) 3767093

Fax (509) 3722610

*Dr. Terrance J. Kavanagh Ph.D.

Research Associate Professor

Department of Environmental Health

University of Washington

4225 Roosevelt Way, NE

Suite 100

Seattle, WA 98105-6099

(206) 6858479

Fax (206) 6854696

*Ms. Patrice Kent

Yakima Indian Nation

Environmental Restoration/Waste Management

Pro.

P.O. Box 151

2808 Main Street

Toppenish, WA 98903

(509) 4522502

Fax (509) 4522503

Mr. Jackson Kinzer

U.S. Department of Energy-Richland Operations

Office

P.O. Box 550, MSIN S7-50

Richland, WA 99352

(509) 767591

Fax (509) 3721215
Ms. Paige Knight

Northwest Environmental Advocates/Hanford

Watch

133 Southwest Second Avenue

Suite 302

Portland, OR 97204-3526

(503) 2950490

Fax (503) 2956634

Mr. Pete Knollmeyer

U.S. Department of Energy

Richland Operations Office

P.O. Box 550, MSIN S7-41

Federal Building, 825 Jadwin Avenue

Richland, WA 99352MSIN

(509) 3767434

Fax (509) 3730205

*Dr. Michael L. Knotek

Associate Laboratory Director

Environmental \& Energy Sciences

Division

Pacific Northwest National Laboratories

MS K1-48

902 Battelle Boulevard

Richland, WA 99352

(509) 3752272

Fax (509) 3756844

*Mr. Kim J. Koegler

Technology Application

Bechtel-Hanford, Inc.

Mail Stop HO-18

3350 George Washington Way

Richland, WA 99352

(509) 3729294

Fax (509) 3729447

Mr. Bruce R. Kowalski

Endowed Professor of Chemistry

Department of Chemistry

University of Washington

Box 351700

Seattle, WA 98195

(206) 5431655

Fax (206) 5436506 
Dr. Martha Krebs

Director

U.S. Department of Energy

Office of Energy Research

Forrestal Building

1000 Independence Avenue, SW

7B-058

Washington, DC 20585

(202) 5865430

Fax (202) 5864120

Fuji Kruder

(503) 9632193

Fax (503) 9635272

*Dr. John P. LaFemina

Manager, Advanced Technology

Development

Environmental Technology Division

Pacific Northwest National Laboratories

P.O. Box 99

3200 Q Avenue

Richland, WA 99352

(509) 3756895

Fax (509) 3754486

*Dr. Jane Long

Head, Environmental Remediation

Technology Earth Sciences Division

Lawrence Berkeley National Labortory

1 Cyclotron Road

Building 90-1116

Berkeley, CA 94720

(510) 4866697

Fax (510) 4864159

Dr. Bill Madia

Lab Director

Pacific Northwest National Laboratories

MS K1-47

Battelle Blvd.

Richland, WA 99352

(509) 3756600

Fax (509) 3756844

Mr. Todd Martin

Hanford Advisory Board

1408 W. Broadway Avenue

Spokane, WA 99201

(509) 3263370

Fax (509) 3262932
Ms. Linda McClain

Assistant Manager

U.S. Department of Energy-Richland

Operations Office

Environmental Restoration

P.0. Box 550, MSIN H0-12

3350 George Washington Way

Richland, WA 99352

(509) 3766628

Fax (509) 3730726

Dr. Melinda L. McClanahan

Associate Director for Science

U.S. Environmental Protection Agency

National Center for Environmental.

Research \& Quality Assurance

401 M Street, SW

MC-8701

Washington, DC 20460

(202) 2605767

Fax (202) 2600929

Dr. Gary McVay

Pacific Northwest National Laboratories

MS K2-45

Battelle Boulevard

Richland, WA 99352

(509) 753762

Fax (509) 3752167

Mr. Toby Michelina

Washington Department of Ecology

Nuclear Waste Program

300 Desmond Drive, SE

Lacey, WA 98503

(360) 4077144

Fax (360) 4077151

*Dr. William Millman

U.S. Department of Energy

ER-14

Germantown, MD 20874

(301) 9035805

Fax (301) 9034110

Mr. Armand Minthom

Confederate Tribes of the Umatilla

Indian Reservation

P.O. Box 638

Pendleton, OR 97801

(503) 278-5211

. Fax (503) 276-3095 
Ms. Marina Moses

U.S. Department of Energy

Office of Science \& Risk Policy

EM - 52, Room 5A - 031

1000 Independence Avenue, SW

Washington, DC 20585

(202) 5867150

Fax (202) 5861492

Mr. John Murphy

U.S. Department of Energy

Richland Operations Office

P.O. Box 550, MSIN H0-12

3350 George Washington Way

Richland, WA 99352

(509) 3767100

Fax (509) 3764360

*Mr. John Neath

U.S. Department of Energy

Richland Operations Office

P.O. Box 550, MSIN K8-50

Richland, WA 99352

(509) 724876

Fax (509) 3724549

*Dr. George (Pinky) Nelson

Associate Vice Provost for Research

University of Washington

P.O. Box 351237

Seattle, WA 98195-1237

(206) 5436616

Fax (206) 6853218

Email pnelson@astro.washington.edu

*Mr. Ralph Patt

Oregon Department of Resources

Water Resources Department

Commerce Building

158-12th Street, NE

Salem, OR 97310

(503) 3783739

Fax (503) 3782496

*Loni M. Peurrung

Senior Research Engineer

Environmental Technology

Pacific Northwest National Laboratory

P.0. Box 999, P7-41

Richland, WA 99352

(509) 3730201

Fax (509) 3721861
*Mr. Lloyd Piper

Assistant Manager, Facility Transition U.S. Department of Energy-Richland

Operations Office

P.O. Box 550, MSIN R3-78

Richland, WA 99352

(509) 3767435

Fax (509) 3739838

Mr. Gerald M. Pollett

Executive Director

Heart of America Northwest

Legal Advocates for Washington

1305 Fourth Avenue

Cobb Building, Suite 208

Seattle, WA 98101

(206) 3821014

Fax (206) 3821148

Ms. Donna Powaukee

Manager

Nez Perce Indian Nation

Environmental Restoration and Waste

Management

P.O. Box 365

Lapwai, ID 83540

(208) 8437375

Fax (208) 8437873

*Mr. Max Power

Nuclear Waste Program Specialist.

Nuclear Waste Program

Washington State Department of

Ecology

P.O. Box 47600

Olympia, WA 98504-7600

(360) 4077118

Fax (360) 4077151

*Ms. Marilyn J. Quadrel

Manager

Risk Management Program Office

Pacific Northwest National Laboratories

P.O. Box 999

3200 Q Avenue, Room 2622

Richland, WA 99352

(509) 3724948

Fax (509) 3754422 
*Dr. Rod K. Quinn

Tank Focus Area Technical Team

Manager

Environmental Technology Division

Pacific Northwest National Laboratories

P.O. Box 999, MSIN K9-69

Battelle Boulevard

Richland, WA 99352

(509) 3756625

Fax (509) 3724662

Ms. Kathie Reed

1019 19th Street, NW

Suite 700

Washington, DC 20036

(202) 6539685

Fax (202) 6535414

*Mr. Gordon J. Rogers

Hanford Advisory Board-Public-at-

Large-Seat

1108 North Road 36

Pasco, WA 99301-2741

(509) 5477403

Fax (509) 5477403

*Mr. Larry Romine

(509) 376-4747

Mr. Eugene Rosolie

Northwest Environmental Advocates

133 SW 2nd Avenue

Suite 302

Portland, OR 97204

(503) 2950490

Fax (503) 2956634

*Mr. Robert M. Rosselli

Assistant Manager for Science \&

Technology

U.S. Department of Energy-Richland

Operations Office

P.O. Box 550, MSIN K8-50

Richland, WA 99352

(509) 3724005

Fax (509) 3724532

*Ms. Shannon Saget

U.S. Department of Energy

(509) 372-4029
*R. Gene Schreckhise

Environmental Science Program

Coordinator

Washington State University at Tri-Cities

100 Sprout Road

Richland, WA 99352

(509) 3727323

- $\operatorname{Fax}(509) 3727471$

*Mr. Doug Sherwood

Hanford Project Manager

Office of Environmental Cleanup

U.S. Environmental Protection Agency

712 Swift Boulevard

Suite 5 .

Richland, WA 99352

(509) 3769529

Fax (509) 3762396

*Dr. Bill D. Shipp

Associate Laboratory Director

Environmental Technology Division

Pacific Northwest National Laboratory

P.O. Box 999

Richland, WA 99352

(509) 3752921

Fax (509) 3724641

Ms. Betsy Silver

U.S. Department of Energy

Idaho Operations Office

850 Energy Drive

MS1219

Idaho Falls, ID 83402

(208) 5269855

Fax (208) 5266249

Mr. Steve Slate

Pacific Northwest National Laboratories

P.O. Box 999, MSIN K9-14

Battelle Boulevard

Richland, WA 99352

(509) 3753903

Fax (509) 3755963 
*Mr. Stanley M. Sobczyk

Environmental Specialist

Environmental Restoration \& Waste

Management

$\mathrm{Nez}$ Perce Indian Nation

P.O. Box 365

Lapwai, ID 83540

(208) 8437375

Fax (208) 8437378

Mr. Gerald C. Sorenson

Program Manager

Batelle (Hanford Advisory Board)

P.O. Box 999

Richland, WA 99352

(509) 3724105

Fax (509) 3765824

Mr. Roger Stanley

Washington Department of Ecology

Nuclear Waste Program

300 Desmond Drive, SE

Lacey, WA 98503

(360) 4077108

Fax (360) 4077151

Ms. Lynne Stembridge

Executive Director

Hanford Education Action League (FEAL)

1720 North Ash

Spokane, WA 99205-4202

(509) 3263370

Fax (509) 3262932

*David Stensel

Professor

Environmental Engineering Program

Department of Civil Engineering

University of Washington

Box 352700 (309 More Hall)

Seattle, WA 98195-2700

(206) 5439358

Fax (206) 6859185
Ms. Betty Tabbutt

Chairperson

Washington Nuclear Waste Advisory

Council

Washington Environmental Council

1063 South Capitol Way

Suite 212

Olympia, WA 98502

(206) 3576548

Fax (206) 9569287

*Dr. Timothy Takaro MD, MPH

Occupational \& Environmental Medicine

University of Washington

Occupational \& Environmental Medicine

Program

Box 354695

Seattle, WA 98104-2499

(206) 6167458

Fax (206) 6164875

*Mr. G. Thomas (Tom) Tebb

TWRS Management Systems Team

Leader

SNF Project Manager

Washington State Departiment of

Ecology

Nuclear Waste Program

1315 W. 4th Avenue

Kennewick, WA 99336-6018

(509) 7363020

Fax (509) 7363030

*Mr. Tom Thornton

Pacific Northwest Laboratories

(509) 373-6668

*Ms. Deborah Trader

Director

Science \& Technology Program Division

U.S. Department of Energy-Richland

Operations Office

P.O. Box 550, MSIN K8-50

Richland, WA 99352

(509) 3724035

Fax (509) 3724549 
*Ms. Nancy Uziemblo

Environmental/Technical Specialist

Nuclear Waste Program, Hanford Project

Washington State Department of

Ecology

1315 West 4th Avenue

Kennewick, WA 99336

(509) 7363014

Fax (509) 7363030

*Mr. John D. Wagoner

Manager

U.S. Department of Energy-Richland

Operations Office

P.0. Box 550, MSIN A7-50

Richland, WA 99352

(509) 3767395

Fax (509) 3764789

*Mr. Jerry D. White

President

Diversified Consulting Resources

201 High Meadows

Richland, WA 99352

(509) 6270473

Fax (509) 6270103

Mr. J.R. Wilkinson

Confederate Tribes of the Umatilla

Indian Reservation

P.O. Box 638

Pendleton, OR 97801

(503) 2760105

Fax (503) 2763095

Mr. Mike Wilson

Washington Department of Ecology

Nuclear Waste Program

300 Desmond Drive, SE

Lacey, WA 98503

(360) 4076702/Fax (360) 4076715
Mr. Don Wodrich

U.S. Department of Energy

Richland Operations Office

P.O. Box 550, MSIN S7-50

Federal Building, 825 Jadwin Avenue

Richland, WA 99352

(509) 3766550

Fax (509) 3762002

*Dr. Tamae Maeda Wong

National Academy of Sciences

2101 Constitution Avenue, NW

Room 273

Washington, DC 20418

(202) 3342156 .

Fax (202) 3342154

Mr. Gerry Woodcock

Westinghouse Hanford Company

P.O. Box 1970 G6-08

Richland, WA 99352

(509) 3767283

Fax (509) 3765999

*Mr. Tom Woods

Yakima Indian Nation

1933 Jadwin Avenue

Suite 110

Richland, WA 99352

(509) 9460101

Fax (509) 9438555

Mr. John Yerxa

U.S. Department of Energy-Richland

Operations Office

Hanford Advisory Board

P.0. Box 550, MSIN A7-75

Richland, WA 99301

(509) 3769678

Fax (509) 3761563 


\title{
Summary of the Environmental Management Science Program Workshop October 9-10, 1996
}

\author{
Oak Ridge, TN
}

\section{Meeting Overview}

On October 9-10, 1996, the Department of Energy (DOE) hosted a meeting in Oak Ridge to discuss the Environmental Management Science Program (EMSP) and the program's role in constructing a site-specific basic research agenda that maps site cleanup needs to basic science areas. Please see the attached list of those who were invited to the workshop.

The meeting opened with an introduction by Rod Nelson, Assistant Manager for Environmental Management, and Johnny Moore, Chairperson of the Site Technology Coordination Group. They emphasized the need for innovative technologies to help meet the goals of the ten year plan, and the importance of basic science research to provide the background necessary to develop these technologies. They further stressed the importance of this workshop, where a diverse group of site representatives, scientists, community stakeholders, and others can help guide the process of turning basic science efforts into better cleanup strategies and technologies.

Drs. Carol Henry, Associate Deputy Assistant Secretary for Science and Risk Policy, Office of Technology Development, Office of Environmental Management (EM) and Robert Marianelli, Director, Chemical Sciences Division, Office of Basic Energy Research, Office of Energy Research (ER), followed with presentations on Environmental Management and Energy Research respectively, and on the joint EMSP. The presentations discussed the history of the program and its experience to date, including the selection of awards for Fiscal Year 1996 (these presentation slides are listed as documents 1 and 2). The purpose of the workshop was threefold, to help the site identify and clarify its basic science needs, to initiate a dialogue between the Oak Ridge problem holders and the local scientific community, and to gain input for the Fiscal Year 1997 EMSP Request for Applications (RFA).

The morning continued with presentations by Suzy Riddle, Division Director for Waste Management, and Bob Sleeman, Division Director for Environmental Restoration. Both presentations highlighted key cleanup challenges and listed basic research needs. Jack Watson, Deputy Coordinator of the Efficient Separations and Processing Crosscutting Program, followed with a discussion on examples of pathways of basic science to technology.

After lunch, Johnny Moore led the group through a document prepared by the Oak Ridge Site Technology Coordination Group (STCG), that laid out 15 technical needs and associated basic science opportunities for the Oak Ridge sites. The group discussed each need in detail and provided the STCG with many suggestions for each. The group also provided a number of suggestions on how best to describe these needs to scientists. 
The morning of the second day opened with a discussion on a proposed format for technical needs statements that was developed by a meeting participant. The rest of the meeting was spent discussing additional site needs, the connection of this program to community stakeholders at the sites, and next steps for Oak Ridge and the EMSP.

These discussions were not intended to result in a consensus among the participants regarding any of the advice given or points raised during the meeting. Further, this summary is not a consensus document. Rather, the points made in this summary were offered by workshop participants who were speaking as individuals, and not formal representatives of their agencies or organizations. This summary tries to capture the major points made during the presentations and meeting discussions and does not attribute comments to any participant. Further, this document is organized by topic area and does not necessarily follow the same chronology of the points raised in the discussion. A list of presentation slides and other documents distributed at the meeting is attached to this summary. These documents are referred to throughout this summary by their number on the list. Copies of these documents are available from The Keystone Center.

\section{General Description of Environmental Issues Facing Oak Ridge}

In setting the context for discussions on Oak Ridge's basic science needs, information about the site, its key environmental threats, and its environmental context was presented. Please refer to the Waste Management and Environmental Restoration presentation slides which are numbered 3 and 4 on the attached document list, for more background. The information provided in these site presentations and in subsequent discussions is summarized below.

- Oak Ridge Operations is responsible for approximately 650 contaminated facilities and release sites, which are located in 14 states.

- The Oak Ridge Reservation is the primary site of concern and is located within the city limits of Oak Ridge, TN, a densely populated urban area.

- There are over 750,000 people within a 50-mile radius of the Oak Ridge plants.

- The Oak Ridge Reservation is located immediately upstream from municipal water intakes and recreational areas.

- Contamination at Oak Ridge poses a threat of exposure to over 15,000 on-site workers.

- Oak Ridge has a diverse legacy of waste burial grounds, some in direct contact with groundwater.

- Oak Ridge, TN is a water rich environment with complex geology of karst and fractured rock that promotes the rapid transport of contaminants in groundwater and surface water.

- Environmental Restoration covers sites in Paducah, KY; Portsmouth, OH; and Oak Ridge, TN.

- see presentation slides for technical needs associated with subsurface contaminants and Decontamination and Decommissioning; and

- Waste Management issues are located primarily in Oak Ridge, TN. 
- see presentation slides for technical needs associated with storage tanks and mixed waste, and for other remaining Environmental Management challenges at Oak Ridge.

\section{Pathways of Basic Science to Technology}

To help workshop participants better conceptualize the role of basic science in meeting cleanup needs, Jack Watson, of the Efficient Separations and Processing Crosscutting Program, gave a presentation on pathways of basic science to technology. (His presentation slides are document 5 on the attached list). This presentation gave several detailed examples of how basic research already has contributed to EM needs and gave several suggestions on how to promote success in the EMSP. Several of his points are summarized below.

- Basic science research already has contributed to EM needs.

-ex. Basic Energy Sciences Program with Crown ether studies, TRUEX, and selective Tc ion exchanger;

- Program continuity and high standards help bring the best investigators to DOE and university laboratories.

- DOE sites need to recognize where EMSP can help the EM mission.

- Research needs must be described adequately and appropriately for EMSP investigators.

- Ideas, information, and needs can start at any point on the technology maturation curve.

- Information should flow both ways between researchers and users.

- Research can be important to users even if it does not yield specific technologies.

- Constant communication is important throughout the research to technology implementation continuum.

- Cultural differences between researchers and users must be understood.

- Identified needs must be translated effectively so they are specific enough to encourage useful research and general enough to encourage ingenuity.

\section{Basic Science Research Needs for the Oak Ridge Sites}

The afternoon of the first day was spent reviewing and discussing the "Basic Science Research Needs for Oak Ridge Sites" document that was developed by the Oak Ridge STCG. This document describes fifteen technical needs, such as inadequate watershed predictive modeling and the need for methods to treat high and low levels of Technetium present in water. It also notes whether these are needed for Environmental Restoration or Waste Management, and describes some of the basic science opportunities associated with the needs, such as developing a basic understanding of the relationship between sediment transport and discharge.

Johnny Moore, Chairperson of the STCG, walked the group through each need and noted comments and suggestions. These comments addressed the content of the needs statements, the formatting of the needs, the language used, the detail used in the descriptions, and other suggestions for making the needs more understandable and enticing to researchers. Members 
of the STCG agreed to incorporate the group's comments into the document, and then send it to the EMSP as the first draft of Oak Ridge's.basic science needs. A copy of the updated list will be forwarded to all meeting participants as soon as it is available to The Keystone Center. Please see document 6 for the original list.

\section{Formatting Basic Science Research Needs Statements}

During discussions on the site's basic science research needs, many different ways to present needs information to scientists were suggested. One workshop participant took these suggestions and developed a strawman format for technical needs statements (listed as document 7). This proposed format was distributed to participants, and discussed in detail. The following points were taken from this discussion.

1. Problem Description

- should relate needs to disciplines and focus on these disciplines

- should describe needs in a way that leaves solutions open, can give examples of solutions being considered

- should describe problems but not be prescriptive in terms of where the research comes from

- should give more background than description

- should give references to literature

- program people need to help others get up to speed with information

- should give references to people, points of contact

- should improve availability of information to researchers

- should set up in a decision tree fashion, provide a short description, then provide a longer, more detailed version if desired

- should make technology needs more prescriptive, what will the results be used for, what are the goals, etc.

- should add how the research is expected to apply in the field and where

2. Contaminants Involved, Medium, Location

- may be necessary increase site-specificity or point out characteristics unique to relevant site(s), such as Oak Ridge's particular geology and hydrogeology

- should describe the location(s) of the problem

- may be necessary to give detailed background information, such as background levels of mercury in area soils

3. Existing Approaches, Window of Opportunity, Related Basic Science Areas

- should give a more specific description of what can be handled and what can not be handled, this would provide more guidance

- should discuss previous efforts and technologies, and explain why these did not work

- should state when treatment must start and when it should be completed

- should provide a description of what the research is trying to improve upon, such as timing, efficiency, cost, etc.

- should explain interim solution in eyes of regulators 
- should not include timing and windows of opportunity because these are fundamentally wrong for basic science

- describing the window of opportunity may discourage research because most needs are short term and results not likely to be available in the shortterm

\section{Reactions and Feedback to the EMSP}

Throughout the meeting, participants provided feedback on the EMSP, and gave a number of suggestions for improving the program to the ER and EM representatives in attendance. These comments and suggestions included the following.

- The EMSP should continue to promote the interaction of investigators and problem holders.

- Pre-proposals are very helpful for primary investigators, particularly because they help researchers figure out what type(s) of research would be useful for users.

- The EMSP should look to needs that will exist five years from now, not just needs that currently exist.

- There is too much money earmarked to the National Labs and National Labs are credited with entire grants even if they subcontract to Universities.

- DOE needs to evaluate the EMSP and its applicability to the sites.

- The EMSP should not assume that research is not useful if it is not directly tied to a focus area or specific technology.

- The EMSP should look into archived work on these issues before investing more money in research.

- The EMSP should include people who are not Federal employees on the relevancy review board.

- EMSP decision-makers should go into the field in order to really understand cleanup problems and to be more anticipatory.

- Research that solidifies current assumptions also is useful.

- The EMSP should allow room for failure -even if research does not work the expected way, it often is still useful.

- The EMSP should put money into determining the state of art in particular areas and identifying where work, gaps, or inefficiencies exist.

- The EMSP should support workshops that bring people together to discuss the state of art in different areas.

- The EMSP should consider using agency-wide announcements instead of RFAs, because they will encourage broader submission and acceptance of ideas and things other than proposals.

\section{Community Involvement}

To address concerns of the community stakeholders present at the meeting, the group spent time discussing representation issues and the role of community stakeholders in the workshop. 
Different ways and reasons to connect the EMSP effort to stakeholders at the sites also were discussed. The following points were taken from these discussions.

- Industry, such as large chemical process industries and consultants in research and development, who are willing to test science applications, were under-represented at the meeting.

- Community involvement is important because stakeholders want to know what is happening and if it makes sense.

- Stakeholder involvement helps educate the community about existing problems.

- Stakeholder inclusion in EMSP meetings helps use limited resources for double-duty.

- Stakeholders take information back to communities and increase public awareness.

- This program will help assure stakeholders that efforts are being made to understand longterm problems.

- This program is good because it shows that DOE is willing to make a commitment to investing in longer-term solutions to problems.

- Inviting stakeholders and sending them information demonstrates openness and allows stakeholders to make their own decisions.

- DOE needs to get information to people who are not actively looking for it; could start by targeting newspapers and television.

- The more communication there is with the public about projects and their purposes, the more public support will be generated.

\section{Next Steps}

The following next steps were identified in the workshop:

- the RFA is due out in November/December;

- the site will put together a good updated draft of needs for the RFA within 2 weeks of the meeting, then will give a refined list to the proposal reviewers; (the updated draft is expected to be completed and sent to EMSP representatives by November 15)

- EMSP wants the site to prioritize its needs;

- participants want copies of the meeting summaries from other sites;

- STCG will be the contact point for future EMSP meetings and other efforts with Oak Ridge;

- the site should take the initiative to find related projects that are already funded and connect users to them; and

- the site wants the EMSP to identify issues, areas, and needs that do not fit into the scope of EMSP, so the site can take action in these areas. 


\title{
Environmental Management Science Program Workshop
}

\author{
October 9-10, 1996 \\ Oak Ridge, Tennesee
}

Participant List

\author{
Ms. Felicia Barnette \\ Research \& Development \\ U.S. Environmental Protection Agency \\ Region 4 \\ 345 Courtland Street \\ Atlanta, GA 30365 \\ 404-347-3555
}

Fax 404-347-1695

Mr. Richard Begley
Consultant
Environmental Management Advisory Board
101 Red Oak Lane
Aiken, SC 29803
803-642-9453

Fax 803-649-2150

Mr. Scott Brooks

Postdoctoral Research Associate

Environmental Sciences Division

ORISE

Oak Ridge National Lab

P.O. Box 2008, MS 6038

Oak Ridge, TN 37831-6038

423-574-6398

Fax 423-576-8646

3sb@ornl.gov

Mr. Gilbert M. Brown

Research Staff

Chemical \& Analytical Sciences Division

Oak Ridge National Laboratory

P.O. Box 2008

Oak Ridge, TN 37831-6119

423-576-2756

Fax 423-574-4939

gbn@ornl.gov
Mr. Robert F. Brown

Consulting Industrial Engineer

East Tennesee Environmental Business

Association

118 Ridgeway Center

Oak Ridge, TN 37830

423-483-0895

Fax 423-482-5396

Dr. Michelle Buchanan

Group Leader

Chemical \& Analytical Sciences Division

Oak Ridge National Laboratory

P.O. Box 2008

Building 5510, Room 121

Oak Ridge, TN 37831-6365

423-574-4868

Fax 423-576-8559

vbu@ornl.gov

Dr. Ariel A. Chialvo

Research Associate Professor

Department of Chemical Engineering

The University of Tennessee

419 Dougherty Engineering Building

Knoxville, TN 37996-2200

423-974-5242

Fax 423-974-4910

achialvo@utk.edu

Mr. Chris D. Cox

Assistant Professor

Department of Civil and Environmental

Engineering

University of Tennessee

223 Perkins Hall

Knoxville, TN 37996-2010

423-974-7729

Fax 423-974-2669

ccox9@utk.edu 
Mr. Allen G. Croff

Oak Ridge National Laboratory, MS-6178

P.O. Box 2008

Oak Ridge, TN 37831-6178

423-574-7192

Fax 423-576-7468

agc@oml.gov

Ms. Sladjana M. Crosley

Chemical Engineer

Oak Ridge National Laboratory

Y-12, Bld. 9204-1, MS 8050

Oak Ridge, TN 37831

423-574-1666

Fax 423-574-0740

crosleysm@ornl.gov

Dr. Peter T. Cummings

Distinguished Professor

Chemical Engineering

University of Tennessee

419 Dougherty Engr.

Knoxville, TN 37996-2200

423-974-0227

Fax 423-974-4910

ptc@utk.edu

Dr. Brian H. Davison Ph.D.

Biochemical Engineering Group Leader

Bioprocessing Research \& Development

Center

Oak Ridge National Laboratory

P.O. Box 2008

Oak Ridge, TN 37831-0226

423-576-8522

Fax 423-574-6442

bod@ornl.gov

Mr. Andrew F. Diefendorf

Technology Application Mánager

Environmental Restoration Program

Oak Ridge National Laboratory

P.O. Box 2008

Building 1509, MS-6400

Oak Ridge, TN 37831-6400

423-241-5997

Fax 423-574-7420

diefendorfs1@ornl.gov
Mr. Stephen Domotor

Office of Science \& Risk Policy (EM-52)

Office of Environmental Management

U.S. Department of Energy

19901 Germantown Road

Germantown, MD 20874-1290

301-903-5053

Fax 301-903-7234

stephen.domotor@em.doe.gov

Mr. Tom Early

DNAPL Product Line Technology Integrator

(SubCon)

Environmental Sciences Division

Oak Ridge National Laboratory

P.O. Box 2008, MS 6400

Oak Ridge, TN 37831-6400

423-576-2103

Fax 423-574-7420

eot@ornl.gov

Mr. David Ernst

Associate Dean

College of Arts and Sciences

Vanderbilt University

311 Kirkland Hall

Nashville, TN 37240

615-343-1483

Fax 615-343-8453

ernst@compsci.cas.vanderbilt.edu

Mr. Richard D. Ferguson

Technical Services Director

Jacobs Engineering Group Inc.

125 Broadway Avenue

Oak Ridge, TN 37830

423-220-4838

Fax 423-220-4848

Dr. Amy Fitzgerald

Executive Director

Oak Ridge Local Oversight Committee, Inc.

(LOC)

136 South Illinois Avenue

Suite 208

Oak Ridge, TN 37830

423-483-1333

Fax 423-482-6572

amyfitz@kormet.org 
Mr. Dimitri Georgopoulos

Project Manager

American Technologies, Inc.

142 Fairbanks Road

Oak Ridge, TN 37830

423-481-4844

Fax 423-481-4850

georgopoulos@earthlink.net

Mr. Mark A Gilbertson

Director of Science \& Risk Policy

Office of Science \& Risk Policy

Office of Science \& Technology

U.S. Department of Energy

Forrestal Building, 1000 Independence Ave., SW

EM-52

Washington, DC 20585

202-586-7150

Fax 202-586-1492

mark.gilbertson@em.doe.gov

Mr. Jim Harless

Environmental Program Manager

Tennessee Department of Environment \&

Conservation (TDEC)

237 Iroquois Road

EX-O. SSAB, Loc, EQAB, etc.

Oak Ridge, TN 37830

423-481-0995

Fax 423-482-1835

Ms. Alicia Harris

Program Manager

ORO-UMTD

HBCU Program Manager

U.S. Department of Energy

3 Main Street

Oak Ridge, TN 37748

423-241-6423

Fax 423-576-5333

qhq@ornl.gov
Dr. Carol J. Henry

Associate Deputy Assistant Secretary

Office of Environmental Management

Office of Science \& Technology

U.S. Department of Energy

Forrestal Building, 1000 Independence Ave., SW

EM-50

Washington, DC 20585

202-586-7150

Fax 202-586-1492

Dr. Roland Hirsch

Acting Director

Medical Applications \& Biophysical

Research Division

Office of Health and Environmental

Research

Office of Energy Research

U.S. Department of Energy

19901 Germantown Road

Germantown, MD 20874-1290

301-903-3213

Fax 301-903-0567

roland.hirsch@oer.doe.gov

Dr. Gary K. Jacobs

Section Head

Earth \& Engineering Sciences

Environmental Sciences Division

Oak Ridge National Laboratory

P.0. Box 2008, Building 1505, MS-6036

Oak Ridge, TN 37831

423-576-0567

Fax 423-576-3989

Dr. Philip M. Jardine

Research Scientist

Environmental Sciences Division

Oak Ridge National Laboratory

P.O. Box 2008

Oak Ridge, TN 37831-6038

423-574-8058

Fax 423-576-8646

ifj@oml.gov 
Dr. Eric N. Kaufman

Research Engineer

Chemical Technology

Oak Ridge National Laboratory

Building 4505 MS 6226

Oak Ridge, TN 37831

423-574-6624

Fax 423-574-6442

ekn@ornl.gov

Ms. Cindy Kendrick

ORO STCG Technical Operations Director

Chemical Technology Division

Lockheed Martin Energy Research

P.O. Box 2001-8620

3 Main Street (37830)

Oak Ridge, TN 37831

423-241-6584

Fax 423-576-5333

cky@ornl.gov

Mr. John Kubarewicz

ORO STCG

Site Technology Coordination Group Buried

Waste Sub Group

Jacobs Engineering Group Inc.

125 Broadway

Oak Ridge, TN 37830

423-220-4943

Fax 423-220-4848

ukf@ornl.gov

Dr. A.P. (Tony) Malinauskas

Director, Environmental Technology

Programs

Lockheed Martin Energy Research

P.O. Box 2008

Oak Ridge, TN 37831-7172

423-576-1092

Fax 423-576-1100

tny@ornl.gov
Dr. Robert Marianelli

Division of Chemical Sciences

Office of Energy Research

U.S. Department of Energy

19901 Germantown Road

Germantown, MD 20874-1290

301-903-5808

Fax 301-903-4110

robert.marianelli@oer.doe.gov

Mr. John F. McCarthy

Environmental Sciences Division

Oak Ridge National Laboratory

P.O. Box 2008

Bethel Valley Road

Oak Ridge, TN 37831-6036

423-576-6606

Fax 423-576-3989

Ms. Maureen M. McDonald

Operations Manager

Oak Ridge Office

Jacobs Engineering Group Inc.

125 Broadway Avenue

Oak Ridge, TN 37830

423-220-4836

Fax 423-220-4848

maureen.mcdonald@jacobs.com

Dr. Robert Mesmer

Section Head

Chemical \& Analytical Sciences Division

Oak Ridge National Laboratory

P.O. Box 2008

Oak Ridge, TN 37831-6110

423-594-4958

Fax 423-574-4961 
Mr. Johnny Moore

Leader, Technology Development \&

Transportation Group

Waste Management \& Technology

Development

Oak Ridge Operations

U.S. Department of Energy

3 Main Street

P.O. Box 2001

Oak Ridge, TN 37831-8620

423-576-3536

Fax 423-576-5333

omo@ornl.gov

Dr. Bruce A. Moyer

Group Leader

Chemical Separations Group

Chemical \& Analytical Sciences Division

Oak Ridge National Laboratory

P.O. Box 2008

4500S, MS-6119

Oak Ridge, TN 37831-6119

423-574-6718

Fax 423-574-4939

moyerba@oml.gov

Mr. David W. Neiswander

Manager, Technology Development

Environmental Restoration, K-25

Lockheed Martin Energy Research

LMES, K-25 Site, P.O. Box 2003

Building K1225, MS 7294

Oak Ridge, TN 37831

423-241-3639

Fax 423-574-6402

neiswanderdw@ornl.gov

Mr. William M. Pardue

Oak Ridge Environmental Management

Site Specific Advisory Boardd

222 Connors Circle

Oak Ridge, TN 37830

423-483-6831

Fax 423-481-8597

76533.226@compuserve.com
Mr. Bob Peelle

Site Specific Advisory Board

130 Oklahoma Avenue

Oak Ridge, TN 37830

423-483-8974

Fax 423-483-8974

Mr. Lal A. Pinnaduwage

Research Staff Member

Health Sciences Research Division

Oak Ridge National Laboratory

P.O. Box 2008

Oak Ridge, TN $\cdot 37831-6122$

423-574-6540

Fax 423-574-6210

llp@oml.gov

Dr. Marvin L. Poutsma

Director

Chemical \& Analytical Sciences Division

Oak Ridge National Laboratory

P.O. Box 2008

Oak Ridge, TN 37831-6129

423-574-5028

Fax 423-574-4902

pou@ornl.gov

Mr. Gregory Reed

Department Head

Department of Civil \& Environmental

Engineering

University of Tennessee

223 Perkins Hall

Knoxville, TN 37996-2010

423-974-2503

Fax 423-974-2669

gdreed@utk.edu

Dr. David E. Reichle

Associate Director

Life Sciences \& Environmental Technologies

Oak Ridge National Laboratory

P.O. Box 2008

Oak Ridge, TN 37831-6253

423-574-4333

Fax 423-574-9869

der@ornl.gov 
Ms. Suzy Riddle

Acting Director

Office of Waste Mangement

U.S. Department of Energy

P.O. Box 2008

Oak Ridge, TN 37831

423-576-9212

Fax 423-576-0785

Dr. Sharon M. Robinson

Section Head, Engineering Development

Section

Chemical Technology Division

Oak Ridge National Laboratory

P.O. Box 2008

Oak Ridge, TN 37831-6044

423-574-6779

Fax 423-576-4195

ssr@ornl.gov

Dr. Gordon Roesler

Office of Environmental Management

Office of Science \& Technology

U.S. Department of Energy

Forrestal Building, 1000 Independence Ave.,

SW

EM-50

Washington, DC 20585

202-586-7150

Fax 202-586-1492

gordon.roesler@em.doe.gov

Dr. John Michael Simonson

Senior Research Staff Member

Chemical \& Avnalytical Sciences Division

Oak Ridge National Laboratory

P.O. Box 2008

Building 4500S, MS-6110

Oak Ridge, TN 37831-6110

423-574-4962

Fax 423-574-4961

simonsonjm@ornl.gov
Mr. Jack Stickney

AIP Supervisor

University of Kentucky Federal Facilities

Oversight Unit

Kentucky Department for Environmental

Protection

P.O. Box 776

Frankfort, KY 40602

502-564-4797

Fax 502-564-5096

jstick@pop.uky.edu

Mr. Harold F. Sturm Jr.

Manager, Environmental Restoration

Technology

Savannah River Technology Center

Savannah River Site

Westinghouse Savannah River Company

Building 773-A, Room A-233

Aiken, SC 29808

803-725-3497

Fax 803-725-8136

harold.sturm@srs.gov

Mr. Tom Treger

U.S. Department of Energy

P.O. Box A

Aiken, SC 29802

803-725-3966

Fax 803-725-7548

Mr. Costas Tsouris

Chemical Engineer

Chemical Technology

Oak Ridge National Laboratory

P.O. Box 2008

Oak Ridge, TN 37831-6226

423-241-3246

Fax 423-241-4829

tsourisc@oml.gov

Mr. Robb Unger

Program Manager

Radian International

1093 Commerce Park Drive

Suite 100

Oak Ridge, TN 37830 


\author{
Dr. Jack Watson \\ Deputy Coordinator \\ Efficient Separation \& Processing Cross- \\ cutting Program \\ Oak Ridge National Laboratory \\ P.O. Box 2008 \\ Oak Ridge, TN 37831-6178 \\ 423-576-6795 \\ Fax 423-576-7468 \\ wat@ornl.gov \\ Mr. Tim Welch \\ Chemical Engineer Staff \\ Chemical Technology Division \\ TFA Pretreatment/ESP \\ Oak Ridge National Laboratory \\ P.0. Box 2008, MS 6273, 4500N \\ Oak Ridge, TN 37922 \\ 423-576-7308 \\ Fax 423-574-7229 \\ welchtd@ornl.gov \\ Mrs. Sylvia Jane Wolfe \\ Research Program Manager, ER-11 \\ Oak Ridge Operations \\ Oak Ridge National Laboratory \\ P.O. Box 2008 \\ Oak Ridge, TN 37831-6269 \\ 423-576-4065 \\ Fax 423-574-9275 \\ wolfesj@ornl.gov
}




\title{
Summary of the Environmental Management Science Program Workshop October 23-24, 1996
}

\author{
Idaho Falls, Idaho
}

\section{Meeting Overview}

On October 23 and 24, 1996, in Idaho Falls, Idaho, approximately 55 individuals representing various interests (see attached Participant List) met to discuss the Department of Energy's (DOE) Environmental Management Science Program (EMSP) and the program's role in constructing a site-specific basic research agenda that maps site cleanup needs to basic science areas. Representatives of the Office of Environmental Management (EM) and the Office of Energy Research (ER), which jointly manage the EMSP, as well as a variety of representatives from the Idaho National Engineering Laboratory (INEL) and other Idaho.DOE offices, were present to convey information on all aspects of the meeting and participate in the ensuing discussions. The Idaho Falls meeting was the fourth in a series of meetings where DOE staff and stakeholders involved with specific DOE sites were able to interact with EMSP representatives.

The participants' discussions, throughout the meeting, were not intended to result in a consensus regarding any of the advice given or points raised during the meeting. Further, this summary is not a consensus document. Rather, the points made in this summary were offered by workshop participants who were speaking as individuals, and not formal representatives of their agencies or organizations. This summary tries to capture the major points made during the meeting. It does not attribute comments to any participant, and does not follow the same chronology of the points raised in the discussion.

\section{Goals of the Meeting}

The goals of the meeting were to exchange information about the EMSP's first year of work and the potential needs of stakeholders; establish better connections between the EMSP and the site and its stakeholders; and assist the EMSP in its efforts to accomplish the mission of identifying and then helping to solve the needs of DOE sites around the country.

\section{Opening Remarks and Presentations}

A series of presentations were given during the first day in an effort to give all participants the context within which the meeting was convened. These presentations are briefly discussed below. Documents developed for each presentation can be obtained from The Keystone Center's Washington, D.C. office by calling Denise Siebert at (202) 783-0248 (see attached list of documents).

Dr. Clay Nichols, Chief Scientist to the Manager, Idaho Operations Office welcomed everyone to the meeting and expressed his pleasure in seeing such interest in the INEL site. His overview of INEL included comments on the history and present day mission of the site, as well as goals for the future. 
Dr. Carol Henry, Associate Deputy Assistant Secretary, Office of Technology, Office of Environmental Management gave a synopsis of the EMSP from the EM perspective. She explained that the EM/ER partnership was formed to develop, administer, and assess the EMSP. The program is being developed in a "needs-based" fashion and therefore EMSP wants to find the specific questions to be answered and problems to be solved as identified by the sites and their stakeholders.

Dr. Michelle Broido, Acting Director, Environmental Sciences Division, Office of Health and Environmental Research, Office of Energy Research presented an overview of the ER efforts associated with the EMSP. She focused on the basic research goal of the EMSP and the hope that this meeting, along with other interactions between EMSP staff and problem holders, would assist in developing an agenda for basic research related to solving site needs. She also related the role that the National Academy of Sciences Committee on Building an EMSP is playing in assisting the EMSP to assess their first year of experience and adapt to the needs of those submitting proposals.

Dr. David Cauffman, Chief Scientist, LMITCO, summarized the INEL experience in connecting research to national needs through two programs: the University Research Consortium (URC) and the Laboratory Directed Research and Development program (LDRD). The URC is intended to, among other things, spark INEL technology development to meet customer needs and build alliances between the INEL, universities, and industry. At the solicitation of proposals stage, the URC requires that the university includes an INEL scientist as a "collaborator." In addition, industrial collaborators are encouraged. The LDRD allows INEL scientists to submit proposals for funding. The collaborator aspect of the URC serves as a bridge between the two programs. Dr. Cauffman also related a series of lessons learned from the programs that were of great interest to the other participants.

Bill Leake, Program Manager, INEL Ten Year Plan, reviewed INEL's Ten Year Plan and its relevance to the DOE vision and mission statement. He then briefly touched upon ways in which the EMSP could possibly assist INEL in meeting its 10 year plan. The presentation also highlighted the difficulty facing INEL in reaching all the goals contained in its 10 year plan.

Jerry Bowman, Assistant Manager, Laboratory Development, Department of Energy's Idaho Office, gave a presentation on INEL's role in technology development and the EMSP. During Mr. Bowman's presentation, Dennis Green, Physical Scientist, Idaho Operations Office, DOE, discussed the specific technology opportunities and needs (gaps) identified by the STCG. Participants followed this discussion by examining two matrices developed by the STCG.

Throughout these presentations a number of questions were raised and brief discussions took place. Many of these issues were deliberated, in further detail, during the facilitated discussion portions of the meeting. The next section of this summary attempts to capture those deliberations. 


\section{INEL Clean-up Opportunities and Needs}

As mentioned above, during the presentations, two overviews of INEL's opportunities and needs were presented to the group. Both efforts were produced by the Site Technology Coordinating Group (STCG). The first was a broad look at the opportunities and needs identified from planned baselines for waste streams where INEL requires clean-up assistance. These included: high level waste, spent fuel, transuranic wastes, and environmental restoration. Common themes across these waste types included characterization and monitoring of the waste form; prediction of future conditions due to physical, chemical, and biological interactions; materials improvement; and separations.

The second was a more in-depth presentation designed to highlight the technological needs and opportunities facing INEL. Needs were defined as specific problems to which no solution currently exists while opportunities were defined as existing technological solutions to which there may well be cheaper, smarter or better solutions. Both the needs and solutions were presented in two matrices which included: prioritization numbers; descriptions of the opportunity or need; internal codes to identify program areas; sources identified to fulfill the need; and existing vulnerabilities. A "potential cost savings" column was developed but not completed.

The general discussion highlighted the fact that the STCG efforts, made prior to this meeting, were driven by technology needs and program managers rather than research scientists.

However, over the next few months, a variety of avenues to include DOE research scientists will be explored. This meeting was the first opportunity; another opportunity exists by taking the lists to the Idaho Research Center to ascertain their basic research needs.

The EMSP staff agreed that these lists are useful in their attempts to write solicitations for research proposals. Participants recommended that all DOE sites meet with their customers to develop prioritized lists of needs. Further, attempting to look beyond the more obvious projects and instead focusing on the high priority needs was suggested as a way to develop basic research projects.

While both the broad overview and the more specific descriptions of opportunities and needs were seen as valuable to certain audiences, the question was raised of whether another level of problem description (between the broad and specific) would be useful. More specifically, would it be useful internally to DOE staff? useful to external research scientists? useful to others?

INEL staff noted that they were in process of developing separate templates of information for each need and opportunity. These will be provided to problem holders who will be asked to assess where their needs are and then INEL will focus on areas where action is not already occurring. Once completed, these templates may serve as a model for other sites to utilize in explaining their needs to research scientists. 
Everyone agreed that the problems need to be described in ways understandable to the research community. Involving the researchers in these types of stakeholder meetings was recognized as one way to assure better understanding.

EMSP staff raised the concern that, during the first year solicitation process, a limited number of nuclear science proposals were received. Participants posited that the budget cuts in the late 1970 s and the resulting exodus from nuclear science programs was one of the major factors in the number of proposals submitted. Participants recommended that DOE commit increased funding for training and resources if they hope to see an increase in nuclear science projects.

\section{Process Suggestions and Issues of Interest Raised During the Meeting}

\section{A. EMSP Solicitation Process}

Participants agreed that the EMSP should be striving to receive the best basic research proposals possible from as wide a range of potential researchers as possible. In order to accomplish those goals, the participants stressed the need to get the solicitations out beyond the legal requirements and to creatively disseminate the information. In addition, the decision-making process must be perceived as fair by all who are considering submitting a proposal, or a number of potentially good basic research projects will not be proposed.

\section{B. Information Needs}

One problem raised by participants related to the lack of information available regarding DOE site needs. A corollary, but no less important, problem was that of ensuring that information gleaned from prior efforts to address the problems is accessible. The templates of information being developed by INEL for each of their needs and opportunities will include information on prior attempts to solve problems. In that way, researchers will have a better idea of what not to try or how to work off of the lessons already learned.

Alternative ways to make existing and future information regarding DOE needs (and prior efforts at solving the problem) readily available include:

- developing Web pages;

- facilitating the use of existing or new reading rooms; and

- developing contact points at each site.

Participants suggested that DOE sites examine the NASA model for developing relationships between academic researchers and internal scientists when developing a contact points program. In essence, each site selects one individual who deals with all external inquiries related to partnering with internal expertise. The "contact point" has a list of all staff at the site, and their expertise, and is able to quickly and efficiently connect them with each other. 


\section{Encouraging Collaboration Between Researchers and Problem Holders}

Participants stressed the potential value of efforts to create partnerships between researchers and problem holders, and industry in some cases, from the beginning of a project. Such collaborative efforts can assist all parties in overcoming some of the problems raised elsewhere in this summary, specifically the disconnect between research and implementation. In addition to users and researchers, it is important to involve regulators and other stakeholders in search for alternative solutions.

In the URC program, discussed by Dr. Cauffman, partnerships between researchers and INEL scientists are required for a proposal to be submitted (in instances where researchers cannot find a partner INEL assigns one). In addition, the program encourages partnerships with industry representatives whenever possible. Industry support ranges from limited to extensive and is usually a promise to assist in future work if the research is successful.

Participants also encouraged the EMSP, as well as the DOE sites, to think about problems in broad ways so that additional relevant scientific fields and their research communities are able to tap into the solicitation process. Specific suggestions included the relationship of numerous academic acid rain studies and U.S. Department of Agriculture studies on fertilizers to soil needs.

\section{Lessons Learned From URC and LDRD Efforts:}

There was general agreement and interest in the list of "lessons learned" from the URD and LDRD efforts which are listed below:

- cultural barriers are not overcome without effort;

- "over the transom" technology transfer does not work well;

- build in desired partnerships from the outset (encourage collaboration between researchers and problem holders);

- technical excellence and applicability to needs are simultaneously achievable;

- planning, expectations management, and incentives help;

- careful identification of needs is important;

- those with needs do not necessarily have imagination; and

- team the problem solver and the solution user early.

\section{E. Finding Ways to Convert Basic Research Findings Into Implementable Solutions}

Participants agreed that there is a need to focus, not only on basic research, but also on applying the findings of that research to specific problems. This notion, that many good ideas are lost between research and application, was termed the "valley of death." Many indicated that the lack of funding to bridge the gap between research and application needed to be addressed when making basic research funding decisions. 
EMSP staff noted that using workshops like this one to bring problem holders and basic researchers together in an effort to find out what the site needs are as determined by those who will eventually apply the basic research findings is one way of helping this conversion.. EMSP also is attempting to keep lines of communication open via World Wide Web sites, ongoing contact with problem holders and researchers, and facilitating communication between the problem holders and researchers.

It was also agreed that efforts like those of INEL to develop prioritized lists of opportunities and needs, are helpful tools in attempts to bridge the "Valley of Death." Both INEL's experience, through development of the priority list and the URC and LDRD programs, and expertise, may allow them to play an important role in future attempts to bridge the gap.

F. Encouraging Private Sector Involvement:

Participants discussed the need to develop incentives to ensure private sector involvement in using basic research (e.g., intellectual property rights). Some felt that, while including the private sector in basic research is important, incentives are unnecessary and not the goal of the EMSP. Others, however, felt that private sector involvement could really spur the basic research program along and indicated support for incentives.

\section{Next Steps}

The following next steps were identified in the workshop:

- INEL will finalize, and share with EMSP staff as well as the research community, the templates developed to describe each technological need and opportunity in a manner accessible to the general research community.

- EMSP and INEL staff will stay in touch as the new RFA is developed and INEL further develops its list of needs and opportunities.

- The STCG will be the contact point for future EMSP meetings and other efforts with INEL.

- The Fiscal Year 1997 RFA is due out in late November or December. 


\title{
Linking the DOE's Environmental Management Science Program With INEL Operation's Long-term Science Research Needs
}

October 23-24, 1996

Idaho Falls, Idaho

Participant List

\author{
Dr. C.A. (Tony) Allen \\ Chemical \& Biological SciencesDepartment \\ Manager \\ Applied Engineering Development \\ Laboratory \\ Lockheed Martin Idaho Technologies \\ Company \\ P.O. Box 1625 \\ MS-2208, IRC \\ Idaho Falls, ID 83415 \\ 208-526-0250 \\ Fax 208-526-8541 \\ cky@inel.gov \\ Dr. George Allen \\ Manager \\ Environmental Programs Business Office \\ Sandia National Laboratories \\ MS-0756 \\ P.O. Box 5800 \\ Albuquerque, NM 87185 \\ 505-844-9769 \\ Fax 505-844-0968 \\ Mr. John Belle \\ Staff Scientist \\ INEL \\ Mr. Warren E. Bergholz Jr. \\ Deputy Manager \\ Idaho Operations Office \\ U.S. Department of Energy \\ 850 Energy Drive \\ MS-1203 \\ Idaho Falls, ID 83401-1563 \\ 208-526-2497 \\ Fax 208-526-0542 \\ Mr. Gerald C. Bowman . \\ Idaho Operations Office \\ U.S. Department of Energy \\ 850 Energy Drive \\ Idaho Falls, ID 83401-1563 \\ 208-526-1925 \\ Fax 208-526-0542 \\ AEDL \\ Dr. Michelle S. Broido Ph.D. \\ Acting Director \\ Environmental Sciences Division \\ Office of Health and Environmental \\ Research \\ U.S. Department of Energy \\ 19901 Germantown Road \\ Germantown, MD 20874-1290 \\ 301-903-3281 \\ Fax 301-903-8519 \\ P.O. Box 1625 \\ Idaho Falls, ID 83145 \\ 208-526-1205 \\ Fax 208-526-6802 \\ bmj@inel.gov
}


Dr. Frank Caccavo Jr.

Research Associate

Center for BioFilm Engineering

Montana State University

409 Coblefish Hall

Bozeman, MT 59717

406-994-1814

Fax 406-994-6095

frank.c@eoc.montana.edu

Dr. David P. Cauffman

Lockheed Martin Idaho Technologies

Company

P.0. Box 1625

MS-2214, IRC

Idaho Falls, ID 83415

208-526-4586

Fax 208-526-5327

cauffman@inel.gov

Ms. Tiajuana Cochnauer

Academic Programs manager

Idaho Operations Office

U.S. Department of Energy

850 Energy Drive

MS 1214

Idaho Falls, ID 83401-1563

208-526-9586

Fax 208-526-8789

cochnatm@inel.gov

Dr. Frederick (Rick) Colwell

Advisory Scientist

Biotechnolgies Department

Idaho National Engineering Laboratory

P.O. Box 1625

Idaho Falls, ID 83415-2203

208-526-0097

Fax 208-526-0828

fxc@inel.gov

Dr. Bill Costerton

Director

Center for Biofilm Engineering

Montana State University

Bozeman, MT 59717-0246

406-994-4770

Fax 406-994-6098

bill@erc.montana.edu
Dr. John Crepeau

Assistant Professor

Mechanical Engineering

University of Idaho at Idaho Falls

P.O. Box 50778

Idaho Falls, ID 83405

208-526-5514

Fax 208-526-1442

stf1004@decit.if.uidaho.edu

Dr. Maxine Dakins

Assistant Professor

University of Idaho at Idaho Falls

1776 Science Center Drive

Idaho Falls, ID 83401

208-526-5530

Fax 208-526-1442

medakins@if.uidaho.edu

Mr. Keith Daum

Advisory Scientist

Environmental Restoration Program

Lockheed Martin Idaho Technologies

Company

P.O. Box 1625

Idaho Falls, ID 83145-3921

208-526-8172

Fax 208-526-4373

dau@inel.gov

Dr. Jim Delmore

Science Fellow

AEDL

Lockheed Martin Idaho Technologies

Company

Box 1625

Idaho Falls, ID 83415-2208

208-526-2820

Fax 208-526-8541

jed2@inel.gov 
Dr. Brad Edredge

Assistant Professor

Chemical Engineering

University of Idaho at Idaho Falls

P.O. Box 50778

Idaho Falls, ID 83405

208-526-1130

Fax 208-526-1442

brade@decit.if.uidaho.edu

Dr. Bruce Erdale

Program Manager

Environmental Management Program Office

Environmental Management/Science \&

Technology

Los Alamos National Laboratory

MS-J591

Los Alamos, NM 87545

505-667-5338

Fax 505-665-8118

Ms. Teresa Fryberger

Deputy Director, Environmental

Applications, EMSL

Environmental Molecular Sciences

Laboratory

Pacific Northwest National Laboratories

P.O.Box 999, MS K2-20

Battelle Blvd

Richland, WA 99352

509-3752709

Fax 5093756916

t_fryberger@pnl.gov

Dr. William H. Glaze

Professor \& Chair

Environmental Sciences \& Engineering

University of North Carolina-Chapel Hill

106 Rosehall, Campus Box 7400

Chapel Hill, NC 27599-7400

919-966-1024

Fax 919-966-2583

bill_glaze@unc.edu
Mr. Dennis W. Green

Physical Scientist

Idaho Operations Office

U.S. Department of Energy

785 DoE Place

Idaho Falls, ID 83401

208-526-1367

Fax 208-526-5964

greendw@inel.gov

Mr. Robert Griswold

Field Program Manager

U.S. Department of Energy

P.O. Box 5400

Albuquerque, NM 87185

505-845-5573

Fax 505-845-4883

Dr. Fred S. Gunnerson

Director

University of Idaho at Idaho Falls

1776 Science Center Drive

Idaho Falls, ID 83405

208-526-1388

Fax 208-526-1442

gunnerson@deoit.if.uiảoho.edu

Mrs. Ellie Hamilton

Idaho National Engineering Laboratory

Site Specific Advisory Board

722 Victor Avenue

Chubbuck, ID 83202

208-237-3664

Fax**

elliehamilton@juno.com

Dr. Anthony E. Hechanova

Research Technologist

Harry Reid Center for Environmental Studies

University of Nevada, Las Vegas

4504 S. Maryland Parkway, Box 454009

Las Vegas, NV 89154-4009

702-895-1457

Fax 702-895-3094

hechanova@brc.neyada,edu 
Dr. Carol J. Henry

Associate Deputy Assistant Secretary

Office of Environmental Management

Office of Science \& Technology

U.S. Department of Energy

Forrestal Building, 1000 Independence Ave., SW

EM-50

Washington, DC 20585

202-586-7150

Fax 202-586-1492

Dr. Edwin W. House

Dean

Graduate Studies \& Research

University of Idaho at Idaho Falls

P.O. Box 8025

Pocatello, ID 83209

208-236-2714

Fax 208-236-4529

housedwi@fs.isu.edu

Dr. Martha A. Krebs

Director

Office of Energy Research

U.S. Department of Energy

Foresstal Building 1000 Independence Ave.,

SW

7B-058

Washington, DC 20585

202-586-5430

Fax 202-586-1009

Dr. Paul A. Lessing

Advisory Engineer/Scienuist

Metals \& Ceramics Department

Research Products Directorate

Lockheed Martin Idaho Technologies

Company

P.O. Box 1625

Idaho Falls, ID 83415-2218

208-526-8776

Fax 208-526-0690
Mr. Jerry Lyle

Idaho Operations Office

U.S. Department of Energy

850 Energy Drive

MS-1101

Idaho Falls, ID 83401-1563

208-526-1148

Fax 208-526-5678

Dr. Jerry McCarthy

Manager

Chemical \& Environmental Engineering

Departiment

Applied Engineering Development

Laboratory

Lockheed Martin Idaho Technologies

Company

P.0. Box 1625

MS-3625, EROB

Idaho Falls, ID $83415-3625$

208-526-5947

Fax 208-526-4017

jre@tis.inel.gov

Dr. David L. Miller

Department Manager

Chemical Waste \& Processors

Lockheed Martin Idaho Technologies

Company

P.0. Box 1625

MS-2208, IRC

Idaho Falls, ID 83415-2206

208-526-9052

Fax 208-526-8541

bsh@inel.gov

Dr. Leland "Roy" Mink

Director

Idaho Water Resources Research Institute

University of Idaho at Idaho Falls

106 Morrill Hall

Moscow, ID 83843

208-885-6424

Fax 208-885-6431

minkr@uidaho.edu 
Mr. Derek G. Moore

Director

Chief Engineer

Applied Engineering \& Research Lab

(AEDL)

Lockheed Martin Idaho Technologies

Company

P.O. Box 1625

MS-3760, EROB

Idaho Falls, ID 83415

208-526-4311

Fax 208-526-4313

dgmr@inel.gov

Ms. Patty Natoni

Assistant Coordinator, EM SSAB-INEL

Idaho Operations Office

U.S. Department of Energy

785 DoE Place, MS 1146

Idaho Falls, ID 83401-1563

208-526-0977

Fax 208-526-0553

nationipm@inel.gov

Mr. James D. Navratil

Engineering/Scientific Fellow

Environmental Operations

Lockheed Martin Idaho Technologies

Company

P.O. Box 1625

Idaho Falls, ID 83415-3921

208-526-1779

Fax 208-526-4373

nav@inel.gov

Dr. Alexander Y. Nazarenko

Department of Chemistry \& Biochemistry

Brigham Young University

C100 Benson Science Building

Provo, UT 84602-6042.

801-378-6042

Fax 801-378-5474

alex_nazarenko@byu.edu
Clayton Ogilvie

Public Adminstrator

Office of Infrastructure Management

Idaho Operations Office

U.S. Department of Energy

785 DOE Place

Idaho Falls, ID 83404

208-526-5190

Fax 208-526-9150

ogilvie@inel.gov

Dr. Gordon Ressler

Office of Environmental Management

Office of Science \& Technology

U.S. Department of Energy

Forrestal Building, 1000 Independence Ave.,

SW

EM-50

Washington, DC 20585

202-586-7150

Fax 202-586-1492

Mr. Charles M. Rice .

Chairman

Idaho National Engineering Laboratory

Site Specific Advisory Board

355 West 14th Street

Idaho Falls, ID 83402

208-522-4955

Fax 208-522-3211

Dr. James A. Seydel

Materials Physics Department Manager

AEDL

Idaho National Engineering Laboratory

Lockheed Martin Idaho Technologies

Company

P.O. Box 1625

Idaho Falls, ID 83415-2209

208-526-6000

Fax 208-526-4621

nde@inel.gov 
Dr. Jean're M. Shreeve

Vice President for Research \& Graduate

Studies

University of Idaho at Idaho Falls

111 Morrill Hall

Moscow, ID 8344-3010

208-885-6651

Fax 208-885-6198

jshreeve@udiaho.edu

Dr. Jack Slater

Department Manager

Optical and Plasma Physics

Lockheed Martin Idaho Technologies

Company

P.0. Box 1625

MS-2211, IRC

Idaho Falls, ID 83415

208-526-7544

Fax 208-526-2814

jxs@inel.gov

Mr. Robert (Bob) N. Snelling

Director, Environmental \& Life Sciences

Applied Engineering \& Development

Laboratory (AEDL)

Lockheed Martin Idaho Technologies

Company

P.O. Box 1625

MS-2213, IRC

Idaho Falls, ID 83415-2213

208-526-4597

Fax 208-526-0603

to3@inel.gov

Mr. Robert A. Starck

Environmental Scientist

Idaho Operations Office

U.S. Department of Energy.

850 Energy Drive

MS-1146

Idaho Falls, ID 83401-1563

208-526-1122

Fax 208-526-0553

starckra@inel.gov
Dr. Ben Sternberg

Chair

Mining \& Geological Engineering

Department

University of Arizona

Building \#12

Tucson, AZ 85721

520-621-2439

Fax 520-621-8330

bks@mge.arizona.edu

Mr. Paul Sturman

Research Associate

Center.for BioFilm Engineering

Montana State University

409 Coblefish Hall

Bozeman, MT 59717

406-994-4770

Fax 406-994-6098

paul.s@erc.montana.edu

Mr. Richard A. Suckel

Working Agreement Project Director

Tribal/DoE Programs

Shoshone-Bannock Tribes

P.O. Box 306

Pima Drive

Fort Hall, ID 83203

208-238-3708

Fax 208-237-0797

Mr. John Wilcynski

Manager

Idaho Operations Office

U.S. Department of Energy

850 Energy Drive

MS-1203

Idaho Falls, ID 83401-1563

208-526-5665

Fax 208-526-0542 
Dr. Stephen E. Williams

Department Head

Plant, Soil, and Insect Sciences

University of Wyoming, College of

Agriculture

P.0. Box 3354

University Station

Laramie, WY 82071-3354

307-766-3103

Fax 307-766-5549

sewms@uwvo.edu

The Keystone Center Staff

Robert W. Craig

President

The Keystone Center

P.O. Box 8606

Keystone, CO 80435-7998

970-468-5822

Fax 970-262-0152

rcraig@keystone.org

Kevin S. Curtis

Senior Associate

The Keystone Center

1001 G Street, NW

Suite 430 West

Washington, DC 20001

202-783-0248

Fax 202-783-0328

kcurtis@keystone.org

Paul S. De Morgan

Associate

The Keystone Center

1001 G Street, NW

Suite 430 West

Washington, DC 20001-4545

202-783-0248

Fax 202-783-0328

pdemorgan@keystone.org
Denise A. Siebert

Office Manager

The Keystone Center

1001 G Street, NW

Suite 430 West

Washington, DC 20001

202-783-0248

Fax 202-783-0328

dsiebert@keystone.org

Alyssa A. Wittenborn

Program Assistant

The Keystone Center

1001 G Street, NW

Suite 430 West

Washington, DC 20001

202-783-0248

Fax 202-783-0328

awittenborn@keystone.org

Kira. Inc. Staff

Pam Frank

Kira, Inc.

1555 King Street

Suite 102

Alexandria, VA 22314

202-586-9756

Fax 202-586-4059

Darlene Roberts

Kira, Inc.

1000 Independence Avenue, SW

3H-017, ER-5

Washington, DC 20858

202-586-7170

Fax 202-586-7719 


\section{Linking the DOE's Environmental Management Science Program \\ With INEL Operation's Long-term Science Research Needs}

October 23-24, 1996

Idaho Falls, Idaho

Participant List

\author{
Dr. C.A. (Tony) Allen \\ Chemical \& Biological SciencesDepartment \\ Manager \\ Applied Engineering Development \\ Laboratory \\ Lockheed Martin Idaho Technologies \\ Company \\ P.O. Box 1625 \\ MS-2208, IRC \\ Idaho Falls, ID 83415 \\ 208-526-0250 \\ Fax 208-526-8541 \\ cky@inel.gov \\ Dr. George Allen \\ Manager \\ Environmental Programs Business Office \\ Sandia National Laboratories \\ MS-0756 \\ P.O. Box 5800 \\ Albuquerque, NM 87185 \\ 505-844-9769 \\ Fax 505-844-0968 \\ Mr. John Belle \\ Staff Scientist \\ INEL \\ AEDL \\ P.O. Box 1625 \\ Idaho Falls, ID 83145 \\ 208-526-1205 \\ Fax 208-526-6802 \\ bmj@inel.gov \\ Mr. Warren E. Bergholz Jr. \\ Deputy Manager \\ Idaho Operations Office \\ U.S. Department of Energy \\ 850 Energy Drive \\ MS-1203 \\ Idaho Falls, ID 83401-1563 \\ 208-526-2497 \\ Fax 208-526-0542 \\ Mr. Gerald C. Bowman . \\ Idaho Operations Office \\ U.S. Department of Energy \\ 850 Energy Drive \\ Idaho Falls, ID 83401-1563 \\ 208-526-1925 \\ Fax 208-526-0542 \\ Dr. Michelle S. Broido Ph.D. \\ Acting Director \\ Environmental Sciences Division \\ Office of Health and Environmental \\ Research \\ U.S. Department of Energy \\ 19901 Germantown Road \\ Germantown, MD 20874-1290 \\ 301-903-3281 \\ Fax 301-903-8519
}


Dr. Frank Caccavo Jr.

Research Associate

Center for BioFilm Engineering

Montana State University

409 Coblefish Hall

Bozeman, MT 59717

406-994-1814

Fax 406-994-6095

frank.c@eoc.montana.edu

Dr. David P. Cauffman

Lockheed Martin Idaho Technologies

Company

P.O. Box 1625

MS-2214, IRC

Idaho Falls, ID 83415

208-526-4586

Fax 208-526-5327

cauffman@inel.gov

Ms. Tiajuana Cochnauer

Academic Programs manager

Idaho Operations Office

U.S. Department of Energy

850 Energy Drive

MS 1214

Idaho Falls, ID 83401-1563

208-526-9586

Fax 208-526-8789

cochnatm@inel.gov

Dr. Frederick (Rick) Colwell

Advisory Scientist

Biotechnolgies Department

Idaho National Engineering Laboratory

P.O. Box 1625

Idaho Falls, ID 83415-2203

208-526-0097

Fax 208-526-0828

fxc@inel.gov

Dr. Bill Costerton

Director

Center for Biofilm Engineering

Montana State University

Bozeman, MT 59717-0246

406-994-4770

Fax 406-994-6098

bill@erc.montana.edu
Dr. John Crepeau.

Assistant Professor

Mechanical Engineering

University of Idaho at Idaho Falls

P.O. Box 50778

Idaho Falls, ID 83405

208-526-5514

Fax 208-526-1442

stf1004@decit.if.uidaho.edu

Dr. Maxine Dakins

Assistant Professor

University of Idaho at Idaho Falls

1776 Science Center Drive

Idaho Falls, ID 83401

208-526-5530

Fax 208-526-1442

medakins@if.uidaho.edu

Mr. Keith Daum

Advisory Scientist

Environmental Restoration Program

Lockheed Martin Idaho Technologies

Company

P.O. Box 1625

Idaho Falls, ID 83145-3921

208-526-8172

Fax 208-526-4373

dau@inel.gov

Dr. Jim Delmore

Science Fellow

AEDL

Lockheed Martin Idaho Technologies

Company

Box 1625

Idaho Falls, ID 83415-2208

208-526-2820

Fax 208-526-8541

jed2@inel.gov 
Dr. Brad Edredge

Assistant Professor

Chemical Engineering

University of Idaho at Idaho Falls

P.O. Box 50778

Idaho Falls, ID 83405

208-526-1130

Fax 208-526-1442

brade@decit.if.uidaho.edu

Dr. Bruce Erdale

Program Manager

Environmental Management Program Office

Environmental Management/Science \&

Technology

Los Alamos National Laboratory

MS-J591

Los Alamos, NM 87545

505-667-5338

Fax 505-665-8118

Ms. Teresa Fryberger

Deputy Director, Environmental

Applications, EMSL

Environmental Molecular Sciences

Laboratory

Pacific Northwest National Laboratories

P.O.Box 999, MS K2-20

Battelle Blvd

Richland, WA 99352

509-3752709

Fax 5093756916

t_fryberger@pnl.gov

Dr. William H. Glaze

Professor \& Chair

Environmental Sciences \& Engineering

University of North Carolina-Chapel Hill

106 Rosehall, Campus Box 7400

Chapel Hill, NC 27599-7400

919-966-1024

Fax 919-966-2583

bill_glaze@unc.edu
Mr. Dennis W. Green

Physical Scientist

Idaho Operations Office

U.S. Department of Energy

785 DoE Place

Idaho Falls, ID 83401

208-526-1367

Fax 208-526-5964

greendw@inel.gov

Mr. Robert Griswold

Field Program Manager

U.S. Department of Energy

P.O. Box 5400

Albuquerque, NM 87185

505-845-5573

Fax 505-845-4883

Dr. Fred S. Gunnerson

Director

University of Idaho at Idaho Falls

1776 Science Center Drive

Idaho Falls, ID 83405

208-526-1388

Fax 208-526-1442

gunnerson@deoit.if.uiảoho.edu

Mrs. Ellie Hamilton

Idaho National Engineering Laboratory

Site Specific Advisory Board

722 Victor Avenue

Chubbuck, ID 83202

208-237-3664

Fax **

elliehamilton@juno.com

Dr. Anthony E. Hechanova

Research Technologist

Harry Reid Center for Environmental Studies

University of Nevada, Las Vegas

4504 S. Maryland Parkway, Box 454009

Las Vegas, NV 89154-4009

702-895-1457

Fax 702-895-3094

hechanova@brc.neyada,edu 
Dr. Carol J. Henry

Associate Deputy Assistant Secretary

Office of Environmental Management

Office of Science \& Technology

U.S. Department of Energy

Forrestal Building, 1000 Independence Ave., SW

EM-50

Washington, DC 20585

202-586-7150

Fax 202-586-1492

Dr. Edwin W. House

Dean

Graduate Studies \& Research

University of Idaho at Idaho Falls

P.O. Box 8025

Pocatello, ID 83209

208-236-2714

Fax 208-236-4529

housedwi@fs.isu.edu

Dr. Martha A. Krebs

Director

Office of Energy Research

U.S. Department of Energy

Foresstal Building 1000 Independence Ave.,

SW

7B-058

Washington, DC 20585

202-586-5430

Fax 202-586-1009

Dr. Paul A. Lessing

Advisory Engineer/Scientist

Metals \& Ceramics Department

Research Products Directorate

Lockheed Martin Idaho Technologies

Company

P.O. Box 1625

Idaho Falls, ID 83415-2218

208-526-8776

Fax 208-526-0690
Mr. Jerry Lyle

Idaho Operations Office

U.S. Department of Energy

850 Energy Drive

MS-1101

Idaho Falls, ID 83401-1563

208-526-1148

Fax 208-526-5678

Dr. Jerry McCarthy

Manager

Chemical \& Environmental Engineering

Departiment

Applied Engineering Development

Laboratory

Lockheed Martin Idaho Technologies

Company

P.O. Box 1625

MS-3625, EROB

Idaho Falls, ID $83415-3625$

208-526-5947

Fax 208-526-4017

jre@tis.inel.gov

Dr. David L. Miller

Department Manager

Chemical Waste \& Processors

Lockheed Martin Idaho Technologies

Company

P.O. Box 1625

MS-2208, IRC

Idaho Falls, ID 83415-2206

208-526-9052

Fax 208-526-8541

bsh@inel.gov

Dr. Leland "Roy" Mink

Director

Idaho Water Resources Research Institute

University of Idaho at Idaho Falls

106 Morrill Hall

Moscow, ID 83843

208-885-6424

Fax 208-885-6431

minkr@uidaho.edu 
Mr. Derek G. Moore

Director

Chief Engineer

Applied Engineering \& Research Lab

(ABDL)

Lockheed Martin Idaho Technologies

Company

P.0. Box 1625

MS-3760, EROB

Idaho Falls, ID 83415

208-526-4311

Fax 208-526-4313

dgmr@inel.gov

Ms. Patty Natoni

Assistant Coordinator, EM SSAB-INEL

Idaho Operations Office

U.S. Department of Energy

785 DoE Place, MS 1146

Idaho Falls, ID 83401-1563

208-526-0977

Fax 208-526-0553

nationipm@inel.gov

Mr. James D. Navratil

Engineering/Scientific Fellow

Environmental Operations

Lockheed Martin Idaho Technologies

Company

P.O. Box 1625

Idaho Falls, ID 83415-3921

208-526-1779

Fax 208-526-4373

nav@inel.gov

Dr. Alexander Y. Nazarenko

Department of Chemistry \& Biochemistry

Brigham Young University

C100 Benson Science Building

Provo, UT 84602-6042

801-378-6042

Fax 801-378-5474

alex_nazarenko@byu.edu
Clayton Ogilvie

Public Adminstrator

Office of Infrastructure Management

Idaho Operations Office

U.S. Department of Energy

785 DOE Place

Idaho Falls, ID 83404

208-526-5190

Fax 208-526-9150

ogilvie@inel.gov

Dr. Gordon Ressler

Office of Environmental Management

Office of Science \& Technology

U.S. Department of Energy

Forrestal Building, 1000 Independence Ave.,

SW

EM-50

Washington, DC 20585

202-586-7150

Fax 202-586-1492

Mr. Charles M. Rice

Chairman

Idaho National Engineering Laboratory

Site Specific Advisory Board

355 West 14th Street

Idaho Falls, ID 83402

208-522-4955

Fax 208-522-3211

Dr. James A. Seydel

Materials Physics Department Manager

AEDL

Idaho National Engineering Laboratory

Lockheed Martin Idaho Technologies

Company

P.O. Box 1625

Idaho Falls, ID 83415-2209

208-526-6000

Fax 208-526-4621

nde@inel.gov 
Dr. Jean'ne M. Shreeve

Vice President for Research \& Graduate

Studies

University of Idaho at Idaho Falls

111 Morrill Hall

Moscow, ID 8344-3010

208-885-6651

Fax 208-885-6198

jshreeve@udiaho.edu

Dr. Jack Slater

Department Manager

Optical and Plasma Physics

Lockheed Martin Idaho Technologies

Company

P.O. Box 1625

MS-2211, IRC

Idaho Falls, D 83415

208-526-7544

Fax 208-526-2814

jxs@inel.gov

Mr. Robert (Bob) N. Snelling

Director, Environmental \& Life Sciences

Applied Engineering \& Development

Laboratory (AEDL)

Lockheed Martin Idaho Technologies

Company

P.O. Box 1625

MS-2213, IRC

Idaho Falls, ID 83415-2213

208-526-4597

Fax 208-526-0603

to3@inel.gov

Mr. Robert A. Starck

Environmental Scientist

Idaho Operations Office

U.S. Department of Energy

850 Energy Drive

MS-1146

Idaho Falls, ID 83401-1563

208-526-1122

Fax 208-526-0553

starckra@inel.gov

\author{
Dr. Ben Sternberg \\ Chair \\ Mining \& Geological Engineering \\ Department \\ University of Arizona \\ Building \#12 \\ Tucson, AZ 85721 \\ 520-621-2439 \\ Fax 520-621-8330 \\ bks@mge.arizona.edu \\ Mr. Paul Sturman \\ Research Associate \\ Center.for BioFilm Engineering \\ Montana State University \\ 409 Coblefish Hall \\ Bozeman, MT 59717 \\ 406-994-4770 \\ Fax 406-994-6098 \\ paul.s@erc.montana.edu \\ Mr. Richard A. Suckel \\ Working Agreement Project Director \\ Tribal/DoE Programs \\ Shoshone-Bannock Tribes \\ P.0. Box 306 \\ Pima Drive \\ Fort Hall, ID 83203 \\ 208-238-3708 \\ Fax 208-237-0797 \\ Mr. John Wilcynski \\ Manager \\ Idaho Operations Office \\ U.S. Department of Energy \\ 850 Energy Drive \\ MS-1203 \\ Idaho Falls, ID 83401-1563 \\ 208-526-5665 \\ Fax 208-526-0542
}


Dr. Stephen E. Williams

Department Head

Plant, Soil, and Insect Sciences

University of Wyoming, College of

Agriculture

P.0. Box 3354

University Station

Laramie, WY 82071-3354

307-766-3103

Fax 307-766-5549

sewms@uwvo.edu

The Keystone Center Staff

Robert W. Craig

President

The Keystone Center

P.O. Box 8606

Keystone, CO 80435-7998

970-468-5822

Fax 970-262-0152

rcraig@keystone.org

Kevin S. Curtis

Senior Associate

The Keystone Center

1001 G Street, NW

Suite 430 West

Washington, DC 20001

202-783-0248

Fax 202-783-0328

kcurtis@keystone.org

Paul S. De Morgan

Associate

The Keystone Center

1001 G Street, NW

Suite 430 West

Washington, DC 20001-4545

202-783-0248

Fax 202-783-0328

pdemorgan@keystone.org
Denise A. Siebert

Office Manager

The Keystone Center

1001 G Street, NW

Suite 430 West

Washington, DC 20001

202-783-0248

Fax 202-783-0328

dsiebert@keystone.org

Alyssa A. Wittenborn

Program Assistant

The Keystone Center

1001 G Street, NW

Suite 430 West

Washington, DC 20001

202-783-0248

Fax 202-783-0328

awittenborn@keystone.org

Kira, Inc. Staff

Pam Frank

Kira, Inc.

1555 King Street

Suite 102

Alexandria, VA 22314

202-586-9756

Fax 202-586-4059

Darlene Roberts

Kira, Inc.

1000 Independence Avenue, SW

3H-017, ER-5

Washington, DC 20858

202-586-7170

Fax 202-586-7719 


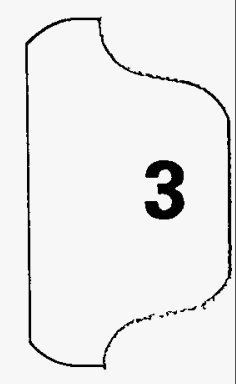


3

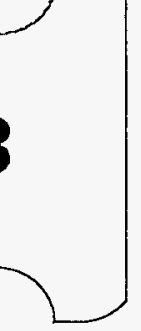




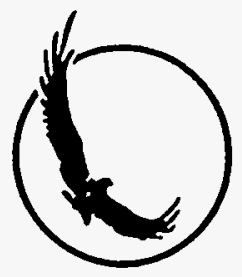

\section{The Keystone Center}

\section{Memorandum}

To: Mark Gilbertson and Marina Moses

From: Kevin Curtis and Alyssa Wittenborn

Subject: $\quad$ Proposal for second-round EMSP meetings

Date: $\quad$ June 20, 1997

In the wake of the recent release of the National Research Council's (NRC) Final Assessment of the Environmental Management Science Program (EMSP), and as the Program moves towards its third round of project funding decisions, timing is right for EMSP leadership to schedule the next round of meetings with the DOE sites and stakeholders that have been involved with the Program to date.

Based upon the experience of The Keystone Center in organizing, convening, and facilitating the first round of EMSP site meetings, we are proposing a process that will result in the convening of a second round of site meetings and potential activities that will build upon and add to last year's meetings and continue to lay the groundwork for future success of the EMSP.

This process would consist of two phases. The first focuses on performing assessment and agenda building activities as the first step in following up the four EMSP site meetings from last year, and makes use of the remaining Fiscal Year 1997 funds. The second proposes a process for convening and following up the four EMSP site meetings and will require the commitment of new funding for Fiscal Year 1998. Proposals and budgets for activities in each of these phases are attached. The objectives of these meetings are described below.

\section{Meeting Objectives}

To honor commitments made by EMSP staff to return to the sites where meetings were held last year and present information on the projects being funded by the Program, follow-up meetings should be convened at Savannah.River, Hanford, Oak Ridge, and Idaho National Engineering and Environment Laboratory. In order to make the second round of meetings as useful as possible to the sites and the EMSP however, these meetings should be designed to achieve several other objectives that will help encourage overall success of the EMSP. 


\section{DRAFT DOCUMENT}

The primary objective of these meetings would be to keep stakeholders informed about the Program's efforts and to continue to improve the communication between the EMSP program staff and the various key stakeholders of the Agency's four largest sites. The expected outcomes of this continued communication are an ever-improving process of identifying the areas in which targeted basic science efforts can most effectively assist the ongoing clean up efforts at the sites. A principal agenda item of each meeting would consist of information on which research areas have been funded by the Program and how the EMSP is addressing the specific research needs identified by each site. As stated in the NRC Final Assessment, stakeholders have legitimate concerns about whether the EMSP is using its financial resources effectively to address important cleanup concerns. In order to continue to build support for the program, these concerns must be addressed. The NRC Final Assessment also suggests that DOE seek to improve and enhance the ways in which it informs potential users of EMSP results about the process and outcome of the proposal selections. The meetings described in this memo would serve these purposes.

Another major objective of the meetings would be to encourage the establishment of stakeholder partnerships. While the NRC report focused upon the EMSP's ability to promote partnerships, it has been clear to Keystone that Assistant Secretary Alm, Director Krebs, the program staff and some site stakeholders all appreciate the catalytic function the EMSP can serve. This is an area in which Keystone believes the second round of meetings can have a tremendously positive impact. We would propose building upon the progress made last year in bringing together diverse stakeholders (i.e. site representatives, national laboratory and university researchers, community stakeholders, regulators, industry, and others) to provide input to the Program and to discuss the ways in which basic science research can help address cleanup needs on their sites.

Success of the EMSP also will rely on efficient transferal and implementation of cleanup technologies that result from EMSP-sponsored research. Sites and stakeholders who remain involved and knowledgeable about the program and feel that the Program has tried to address their concerns will be more likely to support and encourage technology implementation activities. As discussed in the NRC Final Assessment, certain stakeholder groups, particularly industry and government regulators, may be key in encouraging the transfer of research results into cleanup technologies. Inclusion of these groups in the site-specific meetings will help educate them about the EMSP and encourage their commitment to its success.

The following pages detail both the process and budgets proposed to achieve the objectives stated above. 


\title{
Proposal for Assessment and Agenda-building Activities for Four Site-Specific EMSP Meetings
}

\author{
The Keystone Center
}

June 1997-August 1997

\section{Assessment and Agenda-building}

For this phase of the follow-up activities, Keystone staff will utilize the experience and site contacts they gained from last year's EMSP efforts to assure that this year's meetings are designed to most effectively and efficiently meet each site's specific needs regarding the Program. In order to determine the sites' needs, a fairly extensive assessment will be conducted by phone with each of the sites and their stakeholders who participated in last year's activities. Potential information to be gathered includes: what the sites found useful from last year's meetings, what the sites have done to follow-up these efforts, what the sites have heard about the EMSP over the past year, what the sites would like to learn about the EMSP and its efforts to date, who should be involved in these meetings, and which forms of communication about the EMSP are most useful and appropriate for the sites' stakeholders. Specific agendas for each site meeting will be developed and meeting length determined (likely one to two days) based upon this input.

\section{Other Site-specific Activities Designed to Build Understanding of, Support for, the EMSP}

During the assessment period, Keystone staff also will probe the sites and their stakeholders for any other needs they have identified regarding the EMSP. These could include various activities such as a special EMSP workshop for government regulators of the sites, or public outreach efforts on the EMSP that would seek to encourage broad community support for the Program. A list or "menu" of identified site-specific needs and activities will be compiled based upon the advice of those consulted and could be presented at the meetings for further discussion. Based upon the outcomes of these discussions, if additional activities are desired, strategies could then be developed to carry them out.

Expenses associated with the assessment and agenda building phase of the follow-up activities described here will be fully covered by funds remaining in the original DOE-Keystone Center EMSP contract, as detailed in the attached budget. 


\title{
DRAFT DOCUMENT
}

\section{Proposal for Four Site-specific EMSP Meetings}

\author{
The Keystone Center
}

(September-December, 1997

Convening Site-specific Meetings (September - October, 1997)

The second phase of the follow-up activities includes the convening of four site meetings, which will be based upon the information collected during the meeting assessments, and the sitespecific agendas developed with this information (Phase 1). These meetings most likely will be convened during the months of September and October, with two meetings held each month. It is expected that approximately thirty-five participants will attend each meeting along with EMSP and Keystone staff.

Development and Finalization of Meeting Summaries (November-December, 1997)

Following the four site meetings, Keystone staff will develop detailed meeting summaries and will distribute them to participants and $\mathrm{DOE}$ staff for comments. Based upon comments received, these summaries will be finalized and redistributed.

Activities related to convening the meetings and developing the meeting summaries will require a commitment of additional resources from DOE. Exact numbers are provided in the attached budget.

f:269/04/006aaw.doc 


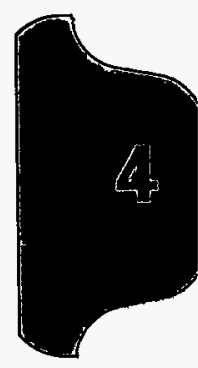




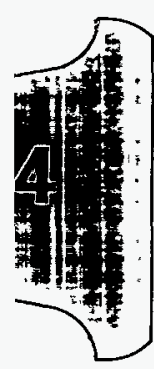




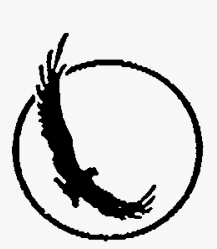

\section{The Keystone Center}

\section{FACSIMILE TRANSMISSION}

To: Mark Gilbertson and Marina Moses

From: Alyssa Wittenborn

Date: October 31, 1997
Fax: 202-586-1492

Fax: $970-262-0152$

Project Number: 269

If you experience problems with this transmission, please contact The Keystone Center at 970-468-5822.

THIS TELEFAX MESSAGE CONTAINS 10 PAGE(S) INCLUDING THIS PAGE.

Per your written request and to fulfill our contractual obligations, attached is a copy of the final report for the EMSP assessments conducted by The Keystone Center over the past few months. Attached to this report, you will find a list of key contacts at each of the site. You also will find reference to participant lists from last year's EMSP meetings. Due to length, these lists will be attached to the hard copy of the report which you will receive by Federal Express on Monday. 
Keystone Science \& Public Policy Program

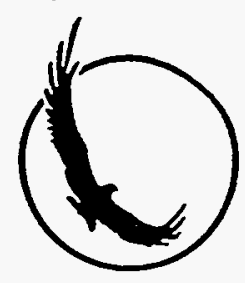

P.O. Box 8606

0175 Summit County Road 8

Keystone, Colorado 80435-7998

Phone 970-468-5822

Fax 970-262-0152

E-Mail: tkcspp@keystone.org
Walter R. Quanstrom

Chairman

Robert W. Craig

President

John R. Ehrmann

Executive Vice President

Michael T. Lesnick. Ph.D.

Senior Vice President and Director

\section{The Keystone Center}

\section{Memorandum}

To: $\quad$ Mark Gilbertson and Marina Moses

From: $\quad$ Kevin Curtis and Alyssa Wittenborn

Subject: $\quad$ Final Report on Assessment Activities for EMSP

Date: $\quad$ October 31,1997

This memorandum is intended to serve as a report of The Keystone Center's findings regarding Environmental Management Science Program (EMSP) follow-up activities at four DOE sites: Savannah River, Oak Ridge, Hanford, and Idaho National Engineering and Environmental Laboratory (INEEL). This information was gathered during assessments which were conducted with the sites during August, September, and October 1997, as well as over the course of The Keystone Center's involvement and experience with the EMSP over the past year and a half.

\section{Assessment Methodology}

Assessments were performed to determine the level of involvement and interest in the EMSP at the four sites, particularly regarding follow-up activities to last year's site-specific EMSP meetings. For each site, Keystone staff identified key participants from last year's meetings and others who represent a variety of interests in the EMSP. For most of the sites, this included site operations personnel, members of the site technology coordination groups (STCG's), members of the citizens advisory boards and other public stakeholders, researchers, regulators, and others. In coordination with EMSP staff, a list of basic questions were developed to aid in the assessments. These included:

- What activities have occurred at the sites related to the EMSP in the last year;

- What information have the sites received regarding the EMSP in the last year;

- What information would the sites like to receive regarding the EMSP;

- Are the sites interested in pursuing activities to follow up last year's EMSP meetings;

- If so, what would the sites like these activities to entail and how should they be structured;

- Who should be involved in these activities; and

- When should these activities occur. 
The following report synthesizes the information collected from these calls. This first part of the report is organized according to a framework for organizing future activities that emerged during the assessments. The rest of the report is organized by site.

\section{Key Conclusions and Framework for Site Follow-up Activities}

During many of the assessment calls, it became apparent that there are three basic categories of interests in the EMSP from the site perspective: external public stakeholders, potential principal investigators (PI's), and technology developers and deployers. These interests exist at each of the sites, and therefore, this section applies to all four of the sites. Based upon the different interests expressed by these groups, we propose they be separated in future follow-up activities or at least that potential follow-up activities recognize the differences between these groups and their needs and interests. Following are general descriptions of each interest category and recommendations specific to each of the four sites under these categories.

\section{External Public Stakeholders:}

Typically, this group consists of members of the citizens advisory boards (CAB's)/site specific advisory boards (SSAB's) and other local community organizations that work with the sites. These stakeholders seem to have a general interest in the EMSP, in terms of what it has done to date (including projects funded related to the sites) and where it expects to go in the future. Follow-up activities with these groups should consist of brief presentations on the history and status of the EMSP, and brief overviews of projects funded that may help address cleanup needs at the sites. These presentations should be followed by question and answer periods, to allow stakeholders to ask more specific questions relevant to their sites. These presentations could be linked with $\mathrm{CAB} / \mathrm{SSAB}$ meetings or could be given the night before meetings are held for the technology developer and deployer groups. One advantage of the second option, is that these presentations would provide interested public stakeholders with sufficient background to attend subsequent, more technical EMSP meetings at the sites.

- Savannah River: There were mixed views on whether a public stakeholder meeting should be held at Savannah River. Most contacts were interested in receiving some basic information on the program, but did not feel they had sufficient time or interest to hold a meeting specifically for that purpose. Therefore, we suggest that EMSP staff draft a basic information document for the site's public stakeholders, potentially including a very basic description of projects funded that relate to the site. Based upon reactions to this document, a short meeting could be scheduled to allow stakeholders to ask more specific questions about the program. Alternatively, those who have interest after reading the document could be invited to attend any additional site EMSP meetings that may occur.

- Oak Ridge: Oak Ridge stakeholders expressed interest in participating in a basic meeting on the EMSP and suggested that EMSP staff give them a presentation on the program at one of their currently scheduled meetings. The SSAB meets the first Tuesday of each month from 6:30 to $9: 30$ p.m.

- Hanford: Hanford stakeholders felt it would be useful to separate them out from the other groups at the site for follow-up activities. Specifically, they are interested in knowing what projects have been funded and what changes have occurred in the program based upon 
recommendations from last year's meeting. In terms of participation, it was commonly held that those who attended last year's meeting were the right people to contact for additional activities.

- INEEL: INEEL SSAB members expressed interest in participating in a short meeting on the EMSP such as that described above. There was a general suggestion that special considerations be made with regard to INEEL stakeholders since they are located across a broad geographic area. Specifically, a meeting of this sort should be organized well in advance, and should be held in conjunction with an existing meeting and/or should take advantage of teleconferencing technologies. It also was suggested that this meeting not take place before January of 1998 , due to busy meeting schedules.

\section{Potential Principal Investigators:}

This group consists of potential PI's from the DOE laboratories, universities, and other research institutions associated with the sites. The primary interests of potential PI's in the EMSP consist of what has been funded by the Program so far; what direction if any, the EMSP is heading in the future; what the long-term funding projections are for the Program; and what specific attributes are desired in future proposed projects. Basically, this group wants advice on how to be competitive for future EMSP grants. A meeting of this sort would likely be relatively short (less than a day) and would consist of a presentation by EMSP staff on these issues followed by a question and answer period.

- All sites: Based upon the similar interests of these groups across the sites and universities contacted, it seems that a meeting of this sort should be held on a national level, and not at each site. Holding one national level meeting with PI's would help alleviate tensions that were noted between researchers at the site laboratories and those at universities who must rely on the sites in order to keep up with the EMSP. Suggestions were made that organizers of this meeting look into using technologies such as teleconferencing so that interested researchers can participate easily regardless of where the meeting is held.

\section{Technology Developers and Deployers:}

This group consists of a variety of site and external representatives, potentially including members of the STCGs, representatives of appropriate focus areas and cross-cutting areas, regulators, PI's of projects directly related to sites, and others. A variety of issues have arisen in discussions with these representatives, and it seems that agendas for these meetings would need to be built around the following topics as appropriate:

1. What projects have been funded to date that are relevant to the needs of the sites and how are these expected to contribute to the solution of site cleanup problems. This should be a fairly detailed presentation by EMSP staff and/or PI's of those projects.

2. What is the best way for the sites to interact with the PI's of these projects. There is interest on the part of the sites in being able to discuss specific site needs with the PI's while not overly influencing their research. Site representatives also are interested in being kept up to date on the state of the research.

3. Are there other basic science research needs that have been identified by the sites since last year's meeting, or have any of last year's needs been refined further? 
4. How will research results be transferred, and where appropriate, translated into technologies for the sites. This discussion will need to involve relevant focus area and cross-cutting area representatives in addition to the PI's and end users. Basically, there have been concerns raised in our discussions about the ability of the focus and cross-cutting areas to serve in technology transfer and development roles in the face of budget cuts. Representatives of these groups have expressed interest in receiving specific guidance from the EMSP on which projects they should pursue first (which are the most promising for the sites) based upon the belief that there will not be sufficient resources to pursue the results from all EMSP projects. There also is interest in linking EMSP research with research being pursued in the focus areas and cross-cutting areas in order to share information and reduce overlap.

- Savannah River: Though Savannah River has not focused specifically on basic science needs since last year's meeting, there was strong interest at the site for holding a meeting to discuss the status and progress of the EMSP, and projects related to Savannah River needs (Questions 1-3). We believe, however, that a discussion related to the issues of research results development and transfer (question 4) would be premature at this time.

- Oak Ridge: Oak Ridge is interested in holding a meeting that addresses all of the questions detailed above (Questions 1-4). Similar to Savannah River, Oak Ridge has not made much progress related to basic science needs in the past year, but there seems to be sufficient interest on the part of the site (primarily due to the involvement of focus area and crosscutting area staff in last year's meeting) to have a broader discussion including the issues of research results development and implementation.

- Hanford: Hanford seems very interested in having a follow-up meeting where EMSP staff present information to the STCG on the Program and the projects funded by EMSP that relate directly to Hanford needs (Questions 1-3). At this meeting, PI's of these projects or members of their staff should be available to discuss these projects with site engineers and others responsible for these problems. This meeting could take place as part of an existing STCG meeting, which are scheduled the third Wednesday of each month. In terms of conducting a meeting to discuss issues related research results development and transfer (question 4), site contacts felt that the site is not currently ready for a meeting of this sort, but that it would be very useful at some point in the future. It was noted that for political reasons, public stakeholders should be informed about any meetings that take place at Hanford, whether or not they are directly involved.

- INEEL: INEEL contacts believe their site has developed a strong communication strategy with the EMSP, and that a meeting to discuss general EMSP issues and specific projects funded by EMSP is not currently necessary. Specifically noted, were the site's recent efforts to further develop their básic science needs list and the site having received a detailed and informative document that lists the projects funded by the program and how they relate to site needs. Similar to Hanford, however, contacts at INEEL felt that a meeting to look into research results development, transfer, and implementation issues (Question 4) would be useful sometime next year. 


\section{Site-specific Information}

While some of the participants contacted in the assessments were enthusiastic about follow-up activities, a number of those contacted would not return our calls. Unfortunately, many of the people who did not return our calls at each of the sites were critical in setting up last year's meetings. In addition; based upon this low response rate, the following information may not be fully representative of site interests and concerns, though information we did receive was fairly consistent across the sites.

\section{Savannah River:}

Most of those contacted at Savannah River had not heard anything specific about the EMSP since last year's meeting. While some were vaguely familiar with the projects funded by the Program, others had not received any information on this. Additional pieces of information requested by the site contacts included: projected funding and expected longevity of the EMSP, how the EMSP will help the site proceed with cleanup, and how staff changes within the EMSP will affect the program. No activities related to the EMSP or basic science needs have taken place at Savannah River since the June 1996 meeting, though the STCG has progressed in identifying and refining technology needs. Contacts felt that participants of last year's meeting were the right people to involve in additional activities.

\section{Oak Ridge:}

Similar to Savannah River, those contacted at Oak Ridge have received little, if any, information regarding the EMSP in the last year. Information requested was also similar to that at Savannah River, with the addition of more detailed information on the projects funded. There was a concern that project abstracts on the EMSP web page are not sufficient or are too science-based to be understandable to interested parties at the site. Oak Ridge has not focused on basic science needs since they revised their needs list subsequent to last October's meeting. In addition, it seems that their STCG has been meeting inconsistently and that their efforts to identify and describe technology needs have recently encountered obstacles. Other comments made regarding last year's meeting were that there were people with significantly different interests artificially mixed together at the meeting, and that several participants felt last year's meeting was a "dog and pony" show for Carol Henry.

Contacts agreed that the participants in last year's meeting were the appropriate people for future activities. It also was noted that based on focus area meetings that are occurring in late November and early December, activities which will require focus area representation should be postponed until after these meetings. The calendar maintained by the Local Oversight Committee (LOC) was reported to be the best source of information available on site events, so more specific information on dates can be obtained from the LOC.

Finally, it was noted that Oak Ridge is currently undergoing significant changes and turmoil. There is a potential for the site to lose a significant percentage of their employees based on appropriations, and the site is also currently looking for a new site contractor to take over in April 1998. These factors must be considered in planning or scheduling any activities at Oak Ridge. 


\section{Hanford:}

Activities related to the EMSP have progressed much further at Hanford in the past year. The Hanford STCG has been working on developing a basic science needs list with site-wide input from DOE, the SSAB, and site contractors. They are interested in knowing when this information would be most useful for EMSP to receive. It also was noted that an EMAB review of the EMSP at Hanford will take place this fall. In general, there was broad interest in the site better connecting with the scientists performing the site-related research.

\section{INEEL:}

INEEL also has made significant progress regarding basic science needs identification and communication at the site. The site currently is working on a science needs list that they expect will be finalized by the STCG in November. Once this happens, the site will submit the list to Kevin Price at headquarters.

Several specific concerns arose in the INEEL assessment. First, many potential PI's in Universities are frustrated because they feel they do not have equal access to literature on previous research conducted by DOE on EMSP issues. Therefore, researchers outside of the site laboratories feel that they are at a disadvantage with regard to performing literature reviews and therefore developing research proposals. A second concern noted was that no minority institutions were selected in the most recent round of EMSP funding, and that only one was selected in the previous round. Finally, as noted above, there were many concerns expressed with regard to remote location issues. Based on these location concerns, it was suggested that any follow-up activities planned with INEEL should creatively try to involve participants.

\section{General Conclusions}

Based upon the information gathered during these assessments, it appears that there is sufficient interest to warrant pursuing some follow-up activities at the sites. Also based upon this information, we believe these activities should follow those described in the "Key Conclusions and Framework for follow-up site activities" section of this report.

In general, there are several key points we would like to get across regarding any follow-up activities held at the sites.

- First, based upon reactions to last year's meetings, it is critical that any follow-up meetings are tailored specifically to the sites and by the sites. The sites should drive these meetings, and should be given primary responsibility for organizing them. In addition, for activities specifically involving the "technology developer and deployer" group, it is critical that the STCGs be heavily involved in specific scheduling and planning.

- Second, at all of the sites except for INEEL, where contacts felt they already have this information, the EMSP should provide detailed presentations on projects funded that relate to site needs. The sites seem to have an idea of what has been funded at their sites, but are generally unaware of projects at other sites and universities that might relate to their problems. The meetings in which these presentations are provided should be attended by the PI's of 
relevant projects or members of their staff who can answer questions and can relate the information in a way that is understandable to everyone in attendance.

- Third, EMSP staff should actively facilitate better connections between EMSP PI's and problem holders, regulators, and other stakeholders. We feel that making and supporting these connections as early as possible will significantly help with the processes of translating, transferring, and implementing the research results at the sites. These connections also will help build additional support for the EMSP and will facilitate acceptance of its results by all involved.

- Fourth, The EMSP continues to hold much promise as a catalyst for better connecting the basic and applied research agendas of the Agency and its four largest sites with the significant environmental cleanup needs facing them. As the EMSP continues to improve its connections with the STCG's and other DOE research efforts, the Program will be laying the groundwork for increased and successful connection and integration of research, technology development and deployment, and cleanup efforts across the complex.

\section{Attachments}

Attached to this report is important contact information for each site that will be useful if follow- $\quad$ up activities are pursued. In addition, the participant lists from each of last year's EMSP meetings are attached. 


\section{Attachment 1: Lists of Key Site Contacts}

The following tables provide the names, positions, and phone numbers of several key people at each of the sites. Based upon our assessments, these are the people who either would be in charge of organizing follow-up activities at the sites or would be able to identify the right people. These lists do not include everyone we contacted during the assessments, nor have we spoken with everyone on these lists. To indicate those with whom we have spoken, we have marked their names with asterisks.

Also, note that each person has either a 1,2, or 3 in front of their name. These numbers are intended to indicate which of the three interest categories these people serve as contacts for: 1 = public stakeholders, 2 = potential PI's, and $3=$ technology developers and deployers. Please see the assessment report for more information on these groups.

\section{Savannah River:}

\begin{tabular}{|l|l|c|}
\hline \multicolumn{1}{|c|}{ Name } & \multicolumn{1}{c|}{ Title/Position } & Phone Number \\
\hline 1. Dawn Haygood & CAB Administrator & $803-952-6971$ \\
\hline 1. *Bill Lawless & CAB member & $706-821-8335$ \\
\hline 2. Dottie Aiken & Georgia Universities & $404-894-2087$ \\
\hline 2. Dr. Walter Castro & South Carolina Universities & $864-656-0954$ \\
\hline 2. *Dr. Nat Frazer & Savannah River Ecology Lab & $803-725-5728$ \\
\hline 3. *Will LaVeille & STCG Chair & $803-725-7663$ \\
\hline 3. Dick Shank & STCG Coordinator & $803-725-8034$ \\
\hline
\end{tabular}

\section{Oak Ridge:}

\begin{tabular}{|l|l|c|}
\hline \multicolumn{1}{|c|}{ Name } & \multicolumn{1}{c|}{ Title/Position } & Phone Number \\
\hline 1. ${ }^{*}$ William Pardue & SSAB member & $423-483-6831$ \\
\hline 1. ${ }^{*}$ Bob Peelle & SSAB member & $423-483-8974$ \\
\hline 1. *Susan Gawarecki & Local Oversight Comm. Director & $423-690-8432$ \\
\hline 2. Alicia Harris & $\begin{array}{l}\text { Historically Black Colleges and } \\
\text {-Universities Pgm Manager }\end{array}$ & $423-241-6423$ \\
\hline 3. ${ }^{*}$ Cindy Kendrick & STCG Coordinator & $423-241-6584$ \\
\hline 3. Johnny Moore & STCG Chair & $423-576-3536$ \\
\hline 3. ${ }^{*}$ Jack Watson & $\begin{array}{l}\text { ORNL Efficient Separation \& } \\
\text { Processing Crosscutting Pgo }\end{array}$ & $423-574-6795$ \\
\hline 3. *Tom Early & $\begin{array}{l}\text { ORNL DNAPL Technology } \\
\text { Integrator (Focus Area) }\end{array}$ & $423-576-2103$ \\
\hline 3. *Sid Jones & $\begin{array}{l}\text { Tennessee Department of } \\
\text { Environment and Conservation }\end{array}$ & $423-481-0995$ \\
\hline
\end{tabular}


Ranford:

\begin{tabular}{|l|l|c|}
\hline \multicolumn{1}{|c|}{ Name } & \multicolumn{1}{|c|}{ Title/Position } & Phone Number \\
\hline 1. *Gordon Rogers & SSAB member, STCG member & $509-547-7403$ \\
\hline 1. *Pam Brown & $\begin{array}{l}\text { Hanford Communities, SSAB \& } \\
\text { STCG member }\end{array}$ & $509-943-7348$ \\
\hline 2. *Dr. Teresa Fryberger & $\begin{array}{l}\text { Environmental Molecular Sciences } \\
\text { Laboratory, PNNL }\end{array}$ & $509-375-2709$ \\
\hline 3. Debbie Trader & Director, STP; STCG member & $509-372-4035$ \\
\hline 3. *Dave Biancosino & $\begin{array}{l}\text { General Engineer, STP; STCG } \\
\text { Subsurface Contamination } \\
\text { Subgroup member }\end{array}$ & $509-372-4084$ \\
\hline 3. *Dr. Bob Julian & $\begin{array}{l}\text { Washington State Department of } \\
\text { Ecology }\end{array}$ & $509-736-5702$ \\
\hline
\end{tabular}

\section{INEEL:}

\begin{tabular}{|l|l|c|}
\hline \multicolumn{1}{|c|}{ Name } & \multicolumn{1}{|c|}{ Title/Position- } & Phone Number \\
\hline 1. Woody Russell & SSAB Coordinator- & $208-526-0561$ \\
\hline 2. *Tiajuana Cochnauer & Academic Programs Manager & $208-526-9586$ \\
\hline 3. Dr. Linda McCoy & $\begin{array}{l}\text { Director, Science and Technology; } \\
\text { EMSP Program Manager for } \\
\text { DOE-ID }\end{array}$ & $208-526-7121$ \\
\hline 3. *Dennis Green & STCG Coordinator & $208-526-1367$ \\
\hline
\end{tabular}

